\title{
Next-to-leading order NMSSM decays with CP-odd Higgs bosons and stops
}

\section{J. Baglio, ${ }^{a}$ C.O. Krauss, ${ }^{b}$ M. Mühlleitner ${ }^{b}$ and K. Walz ${ }^{b}$}

${ }^{a}$ Institute for Theoretical Physics, Tübingen University, Auf der Morgenstelle 14, 72076 Tübingen, Germany

${ }^{b}$ Institute for Theoretical Physics, Karlsruhe Institute of Technology, Wolfgang-Gaede-Str. 1, 76131 Karlsruhe, Germany

E-mail: julien.baglio@uni-tuebingen.de, carla.olivia.k@googlemail.com, margarete.muehlleitner@kit.edu, kathrin.walz@kit.edu

ABSTRACT: We compute the full next-to-leading order supersymmetric (SUSY) electroweak (EW) and SUSY-QCD corrections to the decays of CP-odd NMSSM Higgs bosons into stop pairs. In our numerical analysis we also present the decay of the heavier stop into the lighter stop and an NMSSM CP-odd Higgs boson. Both the EW and the SUSY-QCD corrections are found to be significant and have to be taken into account for a proper prediction of the decay widths.

KeYwORDS: NLO Computations, Supersymmetry Phenomenology

ARXIV EPRINT: 1505.07125 


\section{Contents}

1 Introduction 1

2 The NMSSM Higgs sector 3

3 The tree-level decay width 4

4 SUSY-QCD corrections $\quad 6$

$\begin{array}{lll}5 & \text { The one-loop electroweak corrections } & 11\end{array}$

6 Stop decays at NLO SUSY-QCD and SUSY-EW 15

7 Numerical analysis $\quad 16$

$\begin{array}{lll}7.1 & \text { Pseudoscalar Higgs boson decays into stop pairs } & 17\end{array}$

$\begin{array}{lll}7.2 & \text { Stop decays into a pseudoscalar } & 21\end{array}$

8 Conclusions $\quad 24$

A The loop functions $\quad 24$

\section{Introduction}

The announcement of the discovery of a new boson by the LHC experiments ATLAS $[1,2]$ and CMS [3, 4] has marked a milestone for particle physics. While the properties of this particle are consistent with the Standard Model (SM) predictions, the uncertainties in the experimental data still leave enough room for interpretations in extensions beyond the SM. Among these, supersymmetric (SUSY) models [5-18] certainly belong to the best motivated and most intensely studied ones. In particular the Next-to-Minimal Supersymmetric Extension (NMSSM) [19-33] provides with the introduction of an additional complex superfield $\hat{S}$ a dynamical solution to the $\mu$ problem [34] when the singlet field acquires a non-vanishing vacuum expectation value. Because of new contributions to the quartic coupling $\lambda$, with which $\hat{S}$ couples to the Higgs doublet superfields $\hat{H}_{u}$ and $\hat{H}_{d}$ already present in the Minimal Supersymmetric Extension of the SM (MSSM), the tree-level mass value of the lighter MSSM-like Higgs boson is enhanced. In consequence less important radiative corrections are required to shift the mass value to the measured value of $125 \mathrm{GeV}$ and therefore smaller stop masses and/or mixing are necessary, so that the fine-tuning is reduced [35-47].

After electroweak symmetry breaking (EWSB) the Higgs sector of the NMSSM consists of seven physical Higgs bosons. In the CP-conserving case, which we assume to be valid here, these are three neutral CP-even, two neutral CP-odd and two charged Higgs bosons. The discovery of all Higgs particles is challenging though not impossible at the high-energy option of the LHC. In [48] we investigated the discovery prospects for the NMSSM Higgs 
bosons during the $13 \mathrm{TeV}$ run of the LHC and gave benchmark scenarios that feature Higgs-to-Higgs decays. ${ }^{1}$ If kinematically allowed also decays into supersymmetric particles can become important, as is well known for the MSSM [81, 82]. The one-loop SUSY-QCD corrections to the decays into stops and sbottoms of the MSSM Higgs bosons have been calculated in [83-85] and can change the decay widths by more than $50 \%$, especially near the threshold. The SUSY-QCD corrections have been reanalyzed in [86]. The full electroweak (EW) one-loop corrections to the pseudoscalar decays into squarks have been provided in $[87,88]$ and have turned out to be significant. Equally, the decays of heavy squarks into lighter ones and a Higgs boson can dominate in a wide range of the MSSM parameter space due to the large Yukawa couplings and stop and sbottom mixings [89]. The SUSY-QCD corrections at next-to-leading order (NLO) are of the order of a few ten percent [90] and mostly negative. The one-loop EW corrections to the decays with a pseudoscalar in the final state are significant $[87,88]$. The full one-loop corrections to stop decays and to heavy Higgs decays into sfermions in the complex MSSM have been discussed in [91-93].

The proper interpretation of the experimental data and, once SUSY has been discovered, the aim to pin down the underlying model and distinguish e.g. the NMSSM from the MSSM, require precise predictions both for the parameters of the model and for the observables like e.g. NMSSM Higgs boson production and decay rates [94]. The higher order corrections to the CP-conserving NMSSM Higgs boson masses and self-couplings have been given in [95-105] and [59], respectively. Additionally, there are several codes available for the evaluation of the NMSSM mass spectrum from a user-defined input at a user-defined scale, like NMSSMTools [106-108] which can be interfaced with SOFTSUSY [109, 110], the interface of SARAH [104, 111-114] with SPheno [115, 116], and finally SARAH which has been interfaced with the recently published package FlexibleSUSY [117, 118]. Recently, NMSSMTools has been extended to include also the CP-violating NMSSM [119]. In our Fortran package NMSSMCALC [120] we have included in the CP-conserving and CP-violating NMSSM the full one-loop and the order $\mathcal{O}\left(\alpha_{t} \alpha_{s}\right)$ corrections to the NMSSM Higgs boson masses $[103,121,122]$ and the state-of-the-art higher order corrections to the decays. These include in the CP-conserving case Higgs decays into stops and sbottoms with the SUSY-QCD corrections of [86] which have been adapted from the MSSM to the NMSSM case. Very recently, neutral Higgs production through gluon fusion and bottom-quark annihilation including higher order corrections has been discussed in [123].

With this work we take another step in improving the predictions for the NMSSM Higgs sector. We provide both the NLO SUSY-QCD and the full one-loop EW corrections to the inclusive decay widths of a pseudoscalar NMSSM Higgs boson into stops as well as to the decays of the heavier stop into the lighter one and a pseudoscalar.

The paper is organized as follows. In section 2 we introduce the NMSSM Higgs sector and set our notation. The tree-level decay width of a pseudoscalar Higgs into stops is discussed in section 3, before we present in section 4 the order $\mathcal{O}\left(\alpha_{s}\right)$ SUSY-QCD corrections and in section 5 the one-loop EW corrections. In section 6 we give the results for the SUSY-QCD and -EW corrections to the decays of a heavy stop into a pseudoscalar and a light stop. The numerical analysis is performed in section 7 . We conclude in section 8 .

\footnotetext{
${ }^{1}$ For other recent studies on NMSSM Higgs boson phenomenology, see refs. [46, 47, 49-80].
} 


\section{The NMSSM Higgs sector}

The NMSSM Higgs potential is obtained from the NMSSM superpotential, which we assume to be scale invariant, the soft SUSY breaking terms and the $D$-term contributions, which are the same as in the MSSM. In terms of the superfields $\hat{H}_{u}$ and $\hat{H}_{d}$, coupling to the up- and down-type quarks, respectively, and the singlet superfield $\hat{S}$ the superpotential reads

$$
W_{\mathrm{NMSSM}}=W_{\mathrm{MSSM}}-\epsilon_{i j} \lambda \hat{S} \hat{H}_{d}^{i} \hat{H}_{u}^{j}+\frac{1}{3} \kappa \hat{S}^{3}
$$

where $i, j=1,2$ are the $\mathrm{SU}(2)_{L}$ indices and we have introduced the totally antisymmetric tensor $\epsilon_{i j}$ with $\epsilon_{12}=1$. Working in the CP-invariant NMSSM, the dimensionless parameters $\lambda$ and $\kappa$ are chosen to be real. The MSSM superpotential $W_{\text {MSSM }}$ is given by

$$
W_{\mathrm{MSSM}}=\epsilon_{i j}\left[y_{e} \hat{H}_{d}^{i} \hat{L}^{j} \hat{E}^{c}+y_{d} \hat{H}_{d}^{i} \hat{Q}^{j} \hat{D}^{c}-y_{u} \hat{H}_{u}^{i} \hat{Q}^{j} \hat{U}^{c}\right]
$$

with the quark and lepton superfields and their charge conjugates, indicated by the superscript $c$, denoted by $\hat{Q}, \hat{U}^{c}, \hat{D}^{c}, \hat{L}$ and $\hat{E}^{c}$. The color and generation indices have been suppressed in eq. (2.2). We neglect generation mixing of the quarks, so that the phases of the Yukawa couplings $y_{d}, y_{u}$ and $y_{e}$, which in general are complex, can be reabsorbed by a redefinition of the quark fields. The soft SUSY breaking NMSSM Lagrangian involving the Higgs doublet and singlet component fields $H_{u}, H_{d}$ and $S$ reads

$$
\mathcal{L}_{\text {soft }}=\mathcal{L}_{\text {soft }, \text { MSSM }}-m_{S}^{2}|S|^{2}+\left(\epsilon_{i j} \lambda A_{\lambda} S H_{d}^{i} H_{u}^{j}-\frac{1}{3} \kappa A_{\kappa} S^{3}+\text { h.c. }\right)
$$

with the soft SUSY breaking MSSM Lagrangian

$$
\begin{aligned}
\mathcal{L}_{\text {soft }, \text { MSSM }}= & -m_{H_{d}}^{2} H_{d}^{\dagger} H_{d}-m_{H_{u}}^{2} H_{u}^{\dagger} H_{u}-m_{\tilde{Q}}^{2} \tilde{Q}^{\dagger} \tilde{Q}-m_{\tilde{L}}^{2} \tilde{L}^{\dagger} \tilde{L}-m_{\tilde{U}_{R}}^{2} \tilde{u}_{R}^{*} \tilde{u}_{R}-m_{\tilde{D}_{R}}^{2} \tilde{d}_{R}^{*} \tilde{d}_{R} \\
& -m_{\tilde{E}_{R}}^{2} \tilde{e}_{R}^{*} \tilde{e}_{R}-\left(\epsilon_{i j}\left[y_{e} A_{E} H_{d}^{i} \tilde{L}^{j} \tilde{e}_{R}^{*}+y_{d} A_{D} H_{d}^{i} \tilde{Q}^{j} \tilde{d}_{R}^{*}-y_{u} A_{U} H_{u}^{i} \tilde{Q}^{j} \tilde{u}_{R}^{*}\right]+h . c .\right) \\
& -\frac{1}{2}\left(M_{1} \tilde{B} \tilde{B}+M_{2} \tilde{W}_{k} \tilde{W}_{k}+M_{3} \tilde{G} \tilde{G}+\text { h.c. }\right) .
\end{aligned}
$$

The gaugino fields are denoted by $\tilde{B}, \tilde{W}_{k}(k=1,2,3)$ and $\tilde{G}$, and the left-handed squarks and sleptons are arranged in doublets denoted by $\tilde{Q}=\left(\tilde{u}_{L}, \tilde{d}_{L}\right)^{T}, \tilde{L}=\left(\tilde{\nu}_{L}, \tilde{e}_{L}\right)^{T}$ while the right-handed fields are denoted by $\tilde{u}_{R}, \tilde{d}_{R}$ and $\tilde{e}_{R}$. The soft SUSY breaking mass parameters $m_{X_{(R)}}^{2}$ of the scalar fields $X=S, H_{d}, H_{u}, \tilde{Q}, \tilde{U}, \tilde{D}, \tilde{L}, \tilde{E}$ are real, while the gaugino mass parameters $M_{1}, M_{2}$ and $M_{3}$ and the soft SUSY breaking trilinear couplings $A_{Y}(Y=\lambda, \kappa, U, D, E)$ are in general complex. In the CP-conserving case assumed here, they are, however, real. Again, the respective quark and lepton superfields are understood to refer to all three fermion generations. Note that we have set soft SUSY breaking terms linear and quadratic in the singlet field $S$ to zero.

After expanding the Higgs fields around their vacuum expectation values (VEVs) $v_{u}$, $v_{d}$ and $v_{s}$, chosen to be real and positive,

$$
H_{d}=\left(\begin{array}{c}
\left(v_{d}+h_{d}+i a_{d}\right) / \sqrt{2} \\
h_{d}^{-}
\end{array}\right) \quad H_{u}=\left(\begin{array}{c}
h_{u}^{+} \\
\left(v_{u}+h_{u}+i a_{u}\right) / \sqrt{2}
\end{array}\right) \quad S=\frac{v_{s}+h_{s}+i a_{s}}{\sqrt{2}}
$$


the Higgs mass matrices for the three scalar, two pseudoscalar and the charged Higgs bosons can be derived from the tree-level scalar potential. The mass matrix decomposes into two mass matrices for the $\mathrm{CP}$-even and the $\mathrm{CP}$-odd Higgs fields. The squared $3 \times 3$ mass matrix $M_{S}^{2}$ for the CP-even Higgs fields can be diagonalized through a rotation matrix $\mathcal{R}^{S}$ which yields the CP-even mass eigenstates $H_{i}(i=1,2,3)$ as

$$
\left(H_{1}, H_{2}, H_{3}\right)^{T}=\mathcal{R}^{S}\left(h_{d}, h_{u}, h_{s}\right)^{T} .
$$

The $H_{i}$ are ordered by ascending mass, $M_{H_{1}} \leq M_{H_{2}} \leq M_{H_{3}}$. In order to obtain the CPodd mass eigenstates $A_{1}, A_{2}$ and the massless Goldstone boson $G$ first a rotation $\mathcal{R}^{G}$ to separate $G$ is applied, and then a rotation $\mathcal{R}^{P}$ to obtain the mass eigenstates

$$
\left(A_{1}, A_{2}, G\right)^{T}=\mathcal{R}^{P}\left(a, a_{s}, G\right)^{T}=\mathcal{R}^{P} \mathcal{R}^{G}\left(a_{d}, a_{u}, a_{s}\right)^{T}
$$

which are ordered such that $M_{A_{1}} \leq M_{A_{2}}$.

The minimization of the Higgs potential $V$ requires the terms linear in the Higgs fields to vanish in the vacuum. The corresponding coefficients, which are called tadpoles, therefore have to be zero. The tadpole conditions for the CP-even fields can be exploited to replace $m_{H_{u}}^{2}, m_{H_{d}}^{2}$ and $m_{S}^{2}$ by the tadpole parameters $t_{h_{d}}, t_{h_{u}}$ and $t_{h_{s}}$. Replacing the $\mathrm{SU}(2)_{L}$ and $\mathrm{U}(1)_{Y}$ gauge couplings $g$ and $g^{\prime}$ and the VEVs $v_{u}$ and $v_{d}$ by the electric charge $e$, the gauge boson masses $M_{W}, M_{Z}$ and by $\tan \beta$, the tree-level NMSSM Higgs sector can then be parameterized by the twelve parameters

$$
t_{h_{u}}, t_{h_{d}}, t_{h_{s}}, e, M_{W}^{2}, M_{Z}^{2}, \lambda, \kappa, A_{\lambda}, A_{\kappa}, \tan \beta=\left\langle H_{u}^{0}\right\rangle /\left\langle H_{d}^{0}\right\rangle \quad \text { and } \quad \mu_{\mathrm{eff}}=\lambda\langle S\rangle .
$$

The VEVs of the neutral components of the Higgs fields are denoted by the brackets around the corresponding fields. The sign conventions for $\lambda$ and $\tan \beta$ are chosen such that they are positive. The $\kappa, A_{\lambda}, A_{\kappa}$ and $\mu_{\text {eff }}$ on the other hand can have both signs. Note also, that the parameter $A_{\lambda}$ can be traded for the charged Higgs boson mass $M_{H^{ \pm}}$, which we will do in the following. From now on we will drop the subscript 'eff'. Note that the inclusion of higher order corrections requires also the soft SUSY breaking mass terms for the scalars and the gauginos as well as the trilinear soft SUSY breaking couplings.

\section{The tree-level decay width}

We start by discussing the tree-level decay width of a pseudoscalar Higgs boson $A_{i}(i=1,2)$ into a pair of stops. The stop mass matrix in the interaction basis $\left(\tilde{t}_{L}, \tilde{t}_{R}\right)$ reads

$$
\mathcal{M}_{\tilde{t}}^{2}=\left(\begin{array}{ll}
m_{\mathrm{LL}}^{2} & m_{\mathrm{LR}}^{2} \\
m_{\mathrm{RL}}^{2} & m_{\mathrm{RR}}^{2}
\end{array}\right)
$$

with

$$
\begin{aligned}
m_{\mathrm{LL}}^{2} & =m_{\tilde{Q}}^{2}+m_{t}^{2}+M_{Z}^{2} \cos 2 \beta\left(\frac{1}{2}-\frac{2}{3} \sin ^{2} \theta_{W}\right) \\
m_{\mathrm{RR}}^{2} & =m_{\tilde{t}_{R}}^{2}+m_{t}^{2}+\frac{2}{3} M_{Z}^{2} \cos 2 \beta \sin ^{2} \theta_{W} \\
m_{\mathrm{LR}}^{2}=m_{\mathrm{RL}}^{2} & =m_{t}\left(A_{t}-\mu \cot \beta\right)
\end{aligned}
$$


in terms of the soft SUSY breaking mass parameters $m_{\tilde{Q}}$ and $m_{\tilde{t}_{R}}$, the soft SUSY breaking trilinear coupling $A_{t}$, the higgsino mixing parameter $\mu$, the top and the $Z$ boson masses $m_{t}$ and $M_{Z}$, the mixing angle $\beta$ and the Weinberg angle $\theta_{W}$. The $\mu$ parameter is generated dynamically in the NMSSM and given by

$$
\mu=\frac{\lambda v_{s}}{\sqrt{2}} .
$$

The stop mass matrix is diagonalized by

$$
\mathcal{R}^{\tilde{t}}=\left(\begin{array}{cc}
\cos \theta_{\tilde{t}} & \sin \theta_{\tilde{t}} \\
-\sin \theta_{\tilde{t}} & \cos \theta_{\tilde{t}}
\end{array}\right)
$$

yielding the stop mass eigenstates $\tilde{t}_{i}(i=1,2)$ as

$$
\tilde{t}_{i}=\mathcal{R}_{i s}^{\tilde{t}} \tilde{t}_{s}
$$

where $s=L, R$ and for the squark masses we have $m_{\tilde{t}_{1}}<m_{\tilde{t}_{2}}$ by convention. The mixing angle $\theta_{\tilde{t}}$ and the squark masses are given by

$$
\tan \theta_{\tilde{t}}=\frac{2 m_{\mathrm{LR}}^{2}}{m_{\mathrm{LL}}^{2}-m_{\mathrm{RR}}^{2}-\sqrt{\left(m_{\mathrm{LL}}^{2}-m_{\mathrm{RR}}^{2}\right)^{2}+4 m_{\mathrm{LR}}^{4}}}
$$

and

$$
m_{\tilde{t}_{1,2}}^{2}=\frac{1}{2}\left[m_{\mathrm{LL}}^{2}+m_{\mathrm{RR}}^{2} \mp \sqrt{\left(m_{\mathrm{LL}}^{2}-m_{\mathrm{RR}}^{2}\right)^{2}+4 m_{\mathrm{LR}}^{4}}\right] .
$$

In case of the pseudoscalar only the coupling to two different stop mass eigenstates is non-vanishing. For the tree-level decay width $\Gamma^{\mathrm{LO}}$ we have

$$
\Gamma^{\mathrm{LO}}\left(A_{i} \rightarrow \tilde{t}_{1} \tilde{t}_{2}\right)=\frac{3 \lambda^{1 / 2}\left(M_{A_{i}}^{2}, m_{\tilde{t}_{1}}^{2}, m_{\tilde{t}_{2}}^{2}\right)}{8 \pi M_{A_{i}}^{3}}\left|\sum_{j=1}^{2} \mathbf{Z}_{i j} G_{A_{j}}^{12}\right|^{2}
$$

where $\lambda(x, y, z)=(x-y-z)^{2}-4 y z$ is the two-body phase space function and the coupling $G_{A_{j}}^{12}(j=1,2)$ of the pseudoscalar $A_{j}$ to the stops reads

$$
G_{A_{j}}^{12}=\frac{g m_{t}}{2 M_{W}}\left[\left(\frac{A_{t}}{\tan \beta}+\mu\right) \mathcal{R}_{j 1}^{P}+\frac{\lambda v}{\sqrt{2}} \frac{1}{\tan \beta} \mathcal{R}_{j 2}^{P}\right]
$$

where $v$ is the VEV given by $v^{2}=v_{u}^{2}+v_{d}^{2}$ and $\mathcal{R}_{j k}^{P}$ are the elements of the rotation matrix defined in eq. (2.7). In particular, they are the tree-level mixing matrix elements. In the kinematics of the decay, however, i.e. for the external Higgs field, we use the two-loop corrected Higgs boson masses at order $\mathcal{O}\left(\alpha_{t} \alpha_{s}\right)$, which include the full EW corrections at one-loop order. The renormalization of the Higgs fields and the computation of the mass corrections have been described in refs. [103, 121, 122]. We follow the conventions of these papers, to which we refer the reader for more details. In order to ensure the on-shell properties of the external Higgs field, which in the calculation of the Higgs mass corrections 
has been renormalized in the mixed on-shell- $\overline{\mathrm{DR}}$ scheme, the finite wave function renormalization factors $\mathbf{Z}$ [124] have to be taken into account. The application of the factor $\mathbf{Z}$ to the tree-level matrix $\mathcal{R}^{P}$ (in $G_{A_{j}}^{12}$ ) leads to the rotation matrix $\mathcal{R}^{P, l}$ which, modulo the Goldstone boson $G$, rotates the interaction eigenstates $a$ and $a_{s}$ to the loop corrected mass eigenstates $A_{1}$ and $A_{2}$, cf. [103],

$$
\mathcal{R}_{i l}^{P, l}=\mathbf{Z}_{i j} \mathcal{R}_{j l}^{P} \quad i, j=A_{1}, A_{2}, G \quad l=a, a_{s}, G .
$$

These and the loop-corrected masses are taken from the Fortran code NMSSMCALC [120], in which we choose the on-shell (OS) renormalization for the top/stop sector in order to be in accordance with the renormalization scheme chosen later on both in the SUSY-QCD corrections and in the electroweak corrections.

In eq. (3.10) we have summed over both possible final states $\tilde{t}_{1} \tilde{t}_{2}^{*}$ and $\tilde{t}_{1}^{*} \tilde{t}_{2}$. In the MSSM, i.e. leaving out the singlet contribution $\propto \lambda$ in the coupling $G_{A_{i}}^{12}$, the decay width is proportional to $m_{t}^{2}\left(\mu+A_{t} / \tan \beta\right)^{2} / M_{A_{i}}$. For small values of $\tan \beta$ and not too heavy pseudoscalars, the decay into stops can compete with and even dominate over the decays into top quarks and into charginos and neutralinos. In the NMSSM, this statement has to be taken with caution, however, as the singlet component in the coupling $G_{A_{i}}^{12}$, depending on the scenario, can come with both signs and hence increase or decrease the decay width.

The importance of the pseudoscalar Higgs decays into lighter stops depends on the size of the pseudoscalar Higgs coupling to stop pairs and the available phase space as well as the magnitude of other possible pseudoscalar decay widths. Due to the large amount of parameters entering already the tree-level Higgs sector, the identification of regions in the multidimensional parameter space that lead to large branching ratios is a non-trivial task. In general, the pseudoscalar decays discussed in this paper are important for NMSSM setups with a heavy pseudoscalar, not too heavy stops and where the decays of pseudoscalars into SM particles and electroweakinos are suppressed. Such mass configurations appear in scenarios with large charged Higgs masses and stop sectors with large mixing and/or small soft SUSY breaking stop masses. Furthermore, small values of $\tan \beta$ increase the Higgs couplings to stop pairs. The investigated stop decays on the other hand are interesting for scenarios with large stop mass splitting, not too heavy pseudoscalars and suppressed decays with electroweakinos in the final states. Light pseudoscalars can be realized through small values of $\kappa A_{\kappa}$.

\section{SUSY-QCD corrections}

The SUSY-QCD corrections for the NMSSM pseudoscalar $A_{i}$ decay width differ from the ones of the MSSM [83-85] solely in the tree-level coupling to the stops $G_{A_{i}}^{12}$. We shortly repeat them here for completeness and in order to introduce our renormalization scheme.

The virtual corrections at order $\mathcal{O}\left(\alpha_{s}\right)$ to the pseudoscalar Higgs decays into stops consist of loop diagrams with a gluon, respectively, gluino exchanged in the $A_{i} \tilde{t}_{1} \tilde{t}_{2}$ vertex and of a contribution involving the four-squark vertex, cf. figure 1 (upper). Note that the mixing contributions due to off-diagonal self-energies are absent in the case of pseudoscalar Higgs decays, as $A_{i}$ only couples to different stop mass eigenstates. The computation of 

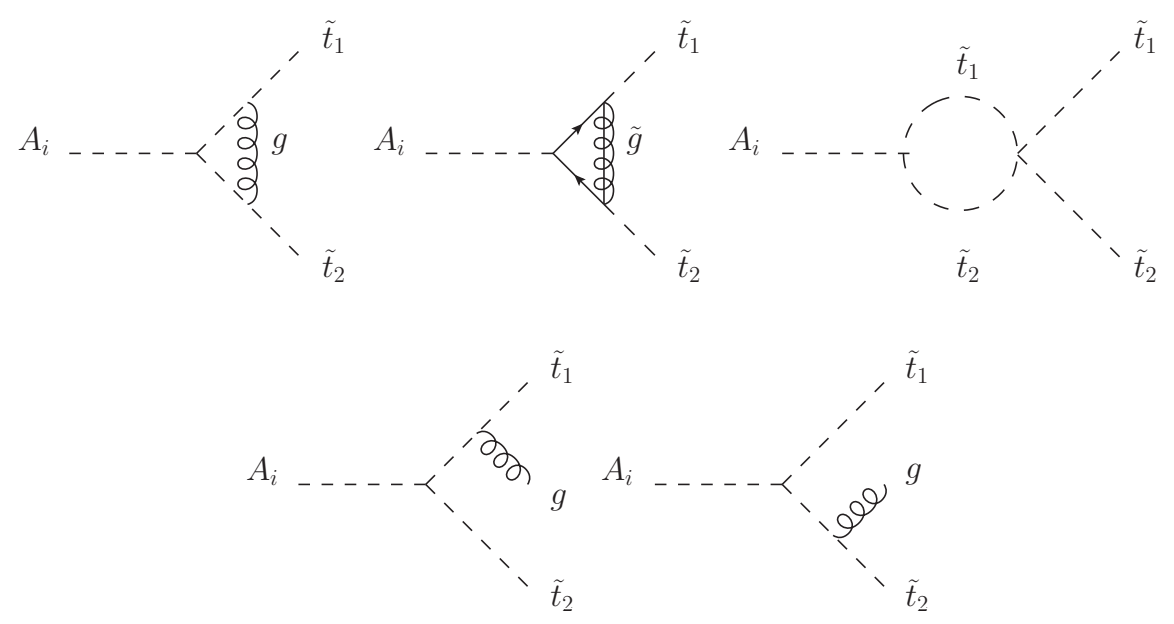

Figure 1. Diagrams contributing at NLO SUSY-QCD to the virtual (upper row) and real corrections (lower row) of the decay $\Gamma\left(A_{i} \rightarrow \tilde{t}_{1} \tilde{t}_{2}\right)$.

the virtual diagrams leads to ultraviolet (UV) and infrared (IR) divergences. We work in dimensional reduction $[125,126]$, which preserves SUSY at the one-loop level. The fields and couplings are then treated in 4 dimensions while the loop integral is performed in $D=4-2 \epsilon$ dimensions. The UV divergences appear as poles in $\epsilon$ and are canceled by the wave function counterterms and the counterterm renormalizing the $A_{i} \tilde{t}_{1} \tilde{t}_{2}$ interaction. The IR divergences are regularized by the introduction of a fictitious gluon mass $\zeta .^{2}$ The IR divergences left over after renormalization are canceled after adding the real corrections. These consist of the radiation of an additional gluon off the final state stops and are shown in figure 1 (lower).

The one-loop corrected decay amplitude can be written as

$$
\Gamma_{\mathrm{QCD}}^{\mathrm{NLO}}=\Gamma^{\mathrm{LO}}+\Gamma_{\mathrm{QCD}}^{(1)}
$$

with

$$
\Gamma_{\mathrm{QCD}}^{(1)}=\operatorname{Re}\left[\frac{\lambda^{1 / 2}\left(M_{A_{i}}^{2}, m_{\tilde{t}_{1}}^{2}, m_{\tilde{t}_{2}}^{2}\right)}{4 \pi M_{A_{i}}^{3}}\left(\sum_{j=1}^{2} \mathbf{Z}_{i j}^{*} G_{A_{j}}^{12 *}\right) \frac{\alpha_{s}}{\pi}\left(\sum_{k=1}^{2} \mathbf{Z}_{i k} \Delta_{A_{k}}^{\mathrm{QCD}}\right)\right]
$$

where again the $\mathbf{Z}$ factors appear to ensure the on-shell properties of the external loopcorrected Higgs field. The $\Delta_{A_{k}}^{\mathrm{QCD}}$ are given by the sum of the virtual, real and counterterm contributions $\Delta_{A_{k}}^{V}, \Delta_{A_{k}}^{R}$ and $\Delta_{A_{k}}^{\mathrm{CT}}$, respectively,

$$
\Delta_{A_{k}}^{\mathrm{QCD}}=\Delta_{A_{k}}^{V}+\Delta_{A_{k}}^{\mathrm{CT}}+\Delta_{A_{k}}^{R} .
$$

From now on a factor $C_{F} \alpha_{s} /(4 \pi)$ with $C_{F}=4 / 3$ is factorized out and already included in eq. (4.2). The virtual corrections receive contributions $\Delta_{A_{k}}^{g}$ from the gluon exchange

\footnotetext{
${ }^{2}$ This is justified as we are working effectively in an Abelian theory, i.e. our calculation does not involve any three-gluon vertex.
} 
diagram, $\Delta_{A_{k}}^{\tilde{g}}$ from the gluino exchange diagram and $\Delta^{4 \tilde{t}}$ from the diagram involving the 4 -squark vertex. They are given by

$$
\begin{aligned}
\Delta_{A_{k}}^{g}= & G_{A_{k}}^{12}\left[B_{0}\left(m_{\tilde{t}_{1}}^{2} ; \zeta, m_{\tilde{t}_{1}}\right)+B_{0}\left(m_{\tilde{t}_{2}}^{2} ; \zeta, m_{\tilde{t}_{2}}\right)-B_{0}\left(M_{A_{k}}^{2} ; m_{\tilde{t}_{1}}, m_{\tilde{t}_{2}}\right)\right. \\
& \left.+2\left(m_{\tilde{t}_{1}}^{2}+m_{\tilde{t}_{2}}^{2}-M_{A_{k}}^{2}\right) C_{0}\left(m_{\tilde{t}_{1}}^{2}, M_{A_{k}}^{2}, m_{\tilde{t}_{2}}^{2} ; \zeta, m_{\tilde{t}_{1}}, m_{\tilde{t}_{2}}\right)\right]
\end{aligned}
$$

for the gluon exchange,

$$
\begin{aligned}
\Delta_{A_{k}}^{\tilde{g}}= & \frac{g m_{t} \mathcal{R}_{k 1}^{P}}{M_{W} \tan \beta}\left[\left(m_{\tilde{g}}-m_{t} \sin \left(2 \theta_{\tilde{t}}\right)\right) B_{0}\left(m_{\tilde{t}_{1}}^{2} ; m_{\tilde{g}}, m_{t}\right)+\left(m_{\tilde{g}}+m_{t} \sin \left(2 \theta_{\tilde{t}}\right)\right) B_{0}\left(m_{\tilde{t}_{2}}^{2} ; m_{\tilde{g}}, m_{t}\right)\right. \\
& \left.-\left(\sin \left(2 \theta_{\tilde{t}}\right) m_{t}\left(m_{\tilde{t}_{1}}^{2}-m_{\tilde{t}_{2}}^{2}\right)+m_{\tilde{g}} M_{A_{k}}^{2}\right) C_{0}\left(m_{\tilde{t}_{1}}^{2}, M_{A_{k}}^{2}, m_{\tilde{t}_{2}}^{2} ; m_{\tilde{g}}, m_{t}, m_{t}\right)\right]
\end{aligned}
$$

for the gluino exchange, and

$$
\Delta_{A_{k}}^{4 \tilde{t}}=G_{A_{k}}^{12} B_{0}\left(M_{A_{k}}^{2} ; m_{\tilde{t}_{1}}, m_{\tilde{t}_{2}}\right)
$$

for the 4-squark vertex diagram, where $B_{0}$ and $C_{0}$ are the Passarino-Veltman scalar twoand three-point functions $[127,128]$, cf. appendix A for their definitions.

The counterterm corrections consist of the renormalization of the external squark wave functions $Z_{\tilde{t}_{j} \tilde{t}_{j}}(j=1,2)$ and the renormalization of the $A_{k} \tilde{t}_{1} \tilde{t}_{2}$ interaction vertex. Note that the wave function renormalization of the pseudoscalar does not contribute at order $\mathcal{O}\left(\alpha_{s}\right)$ and hence to the SUSY QCD corrections. The parameters $\lambda, v, v_{s}, \tan \beta, M_{W}, M_{Z}$ and $e$ are not renormalized by the strong interaction, so that the counterterm reads

$$
\Delta_{A_{k}}^{\mathrm{CT}}=\frac{1}{2} G_{A_{k}}^{12}\left(\delta Z_{\tilde{t}_{1} \tilde{t}_{1}}+\delta Z_{\tilde{t}_{2} \tilde{t}_{2}}\right)+\frac{\partial G_{A_{k}}^{12}}{\partial m_{t}} \delta m_{t}+\frac{\partial G_{A_{k}}^{12}}{\partial A_{t}} \delta A_{t}
$$

leading to

$$
\Delta_{A_{k}}^{\mathrm{CT}}=\frac{1}{2} G_{A_{k}}^{12}\left(\delta Z_{\tilde{t}_{1} \tilde{t}_{1}}+\delta Z_{\tilde{t}_{2} \tilde{t}_{2}}\right)+G_{A_{k}}^{12} \frac{\delta m_{t}}{m_{t}}+\frac{g m_{t}}{2 M_{W}} \frac{\mathcal{R}_{k 1}^{P}}{\tan \beta} \delta A_{t} .
$$

The counterterm $\delta A_{t}$ of the trilinear coupling is given by the quark and squark mass counterterms, $\delta m_{t}$ and $\delta m_{\tilde{t}_{1,2}}$, and the mixing angle counterterm $\delta \theta_{\tilde{t}}$,

$$
\delta A_{t}=\frac{1}{2 m_{t}}\left[\left(m_{\tilde{t}_{1}}^{2}-m_{\tilde{t}_{2}}^{2}\right)\left(2 \cos \left(2 \theta_{\tilde{t}}\right) \delta \theta_{\tilde{t}}-\sin \left(2 \theta_{\tilde{t}}\right) \frac{\delta m_{t}}{m_{t}}\right)+2 \sin \left(2 \theta_{\tilde{t}}\right)\left(m_{\tilde{t}_{1}} \delta m_{\tilde{t}_{1}}-m_{\tilde{t}_{2}} \delta m_{\tilde{t}_{2}}\right)\right] .
$$

In the (s)quark sector we adopt OS renormalization with the quark and squark masses defined as the poles of their respective propagators and the squark wave function renormalization constants defined such that the residues of the poles are equal to one. Defining the following structure for the quark self-energy, where $\mathcal{P}_{L, R}$ denote, respectively, the leftand right-chiral projector,

$$
\Sigma_{t}\left(p^{2}\right) \equiv \not p \Sigma_{t}^{L}\left(p^{2}\right) \mathcal{P}_{L}+\not p \Sigma_{t}^{R}\left(p^{2}\right) \mathcal{P}_{R}+m_{t} \Sigma^{\mathrm{Ls}}\left(p^{2}\right) \mathcal{P}_{L}+m_{t} \Sigma^{\mathrm{Rs}}\left(p^{2}\right) \mathcal{P}_{R}
$$

we have for the top quark mass counterterm

$$
\delta m_{t}=\frac{1}{2} \operatorname{Re}\left(m_{t} \Sigma_{t}^{L}\left(m_{t}^{2}\right)+m_{t} \Sigma_{t}^{R}\left(m_{t}^{2}\right)+\Sigma_{t}^{\mathrm{Ls}}\left(m_{t}^{2}\right)+\Sigma_{t}^{\mathrm{Rs}}\left(m_{t}^{2}\right)\right) .
$$




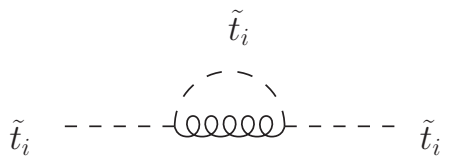

$g$

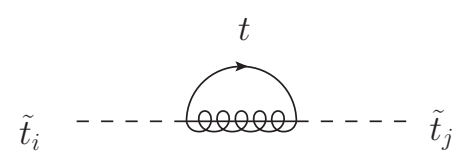

$\tilde{g}$

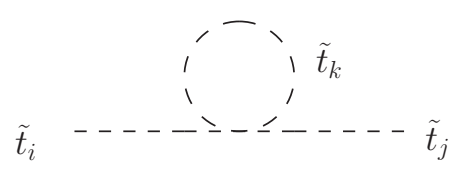

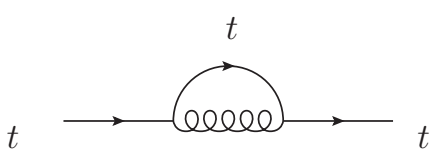

$g$

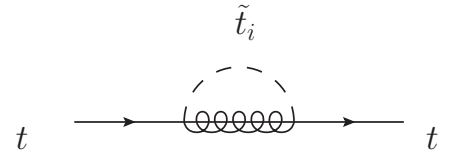

$\tilde{g}$

Figure 2. Diagrams contributing at NLO SUSY-QCD to the squark self-energies (upper row) and to the quark self-energies (lower row).

The squark mass and wave function counterterms are given by $(j=1,2)$

$$
\begin{aligned}
\delta m_{\tilde{t}_{j}}^{2} & =\operatorname{Re} \Sigma_{\tilde{t}_{j} \tilde{t}_{j}}\left(m_{\tilde{t}_{j}}^{2}\right) \\
\delta Z_{\tilde{t}_{j} \tilde{t}_{j}} & =-\left.\operatorname{Re} \frac{\partial \Sigma_{\tilde{t}_{j} \tilde{t}_{j}}\left(p^{2}\right)}{\partial p^{2}}\right|_{p^{2}=m_{\tilde{t}_{j}}^{2}} .
\end{aligned}
$$

In eq. (4.12) the $\Sigma_{\tilde{t}_{j} \tilde{t}_{j}}$ denote the diagonal parts of the squark self-energies. The diagrams contributing at order $\mathcal{O}\left(\alpha_{s}\right)$ to the squark and quark self-energies are depicted in figure 2 (upper) and (lower), respectively.

The mass counterterms read

$$
\begin{aligned}
\frac{\delta m_{t}}{m_{t}}= & -\operatorname{Re}\left[2 B_{1}\left(m_{t}^{2} ; m_{t}, \zeta\right)+B_{1}\left(m_{t}^{2} ; m_{\tilde{g}}, m_{\tilde{t}_{1}}\right)+B_{1}\left(m_{t}^{2} ; m_{\tilde{g}}, m_{\tilde{t}_{2}}\right)+4 B_{0}\left(m_{t}^{2} ; m_{t}, \zeta\right)\right. \\
& \left.+\sin \left(2 \theta_{\tilde{t}}\right) \frac{m_{\tilde{g}}}{m_{t}}\left(B_{0}\left(m_{t}^{2} ; m_{\tilde{g}}, m_{\tilde{t}_{1}}\right)-B_{0}\left(m_{t}^{2} ; m_{\tilde{g}}, m_{\tilde{t}_{2}}\right)\right)\right] \\
m_{\tilde{t}_{1}} \delta m_{\tilde{t}_{1}}= & \operatorname{Re}\left[2 m_{t} m_{\tilde{g}} \sin \left(2 \theta_{\tilde{t}}\right) B_{0}\left(m_{\tilde{t}_{1}}^{2} ; m_{t}, m_{\tilde{g}}\right)+\frac{1}{2}\left[\left(1+\cos ^{2}\left(2 \theta_{\tilde{t}}\right)\right) A_{0}\left(m_{\tilde{t}_{1}}\right)\right.\right. \\
& \left.+\sin ^{2}\left(2 \theta_{\tilde{t}}\right) A_{0}\left(m_{\tilde{t}_{2}}\right)\right]-2 m_{\tilde{t}_{1}}^{2} B_{0}\left(m_{\tilde{t}_{1}}^{2} ; m_{\tilde{t}_{1}}, \zeta\right)-A_{0}\left(m_{\tilde{g}}\right)-A_{0}\left(m_{t}\right) \\
& \left.+\left(m_{\tilde{t}_{1}}^{2}-m_{t}^{2}-m_{\tilde{g}}^{2}\right) B_{0}\left(m_{\tilde{t}_{1}}^{2} ; m_{t}, m_{\tilde{g}}\right)\right] \\
m_{\tilde{t}_{2}} \delta m_{\tilde{t}_{2}}= & \operatorname{Re}\left[-2 m_{t} m_{\tilde{g}} \sin \left(2 \theta_{\tilde{t}}\right) B_{0}\left(m_{\tilde{t}_{2}}^{2} ; m_{t}, m_{\tilde{g}}\right)+\frac{1}{2}\left[\left(1+\cos ^{2}\left(2 \theta_{\tilde{t}}\right)\right) A_{0}\left(m_{\tilde{t}_{2}}\right)\right.\right. \\
& \left.+\sin ^{2}\left(2 \theta_{\tilde{t}}\right) A_{0}\left(m_{\tilde{t}_{1}}\right)\right]-2 m_{\tilde{t}_{2}}^{2} B_{0}\left(m_{\tilde{t}_{2}}^{2} ; m_{\tilde{t}_{2}}, \zeta\right)-A_{0}\left(m_{\tilde{g}}\right)-A_{0}\left(m_{t}\right) \\
& \left.+\left(m_{\tilde{t}_{2}}^{2}-m_{t}^{2}-m_{\tilde{g}}^{2}\right) B_{0}\left(m_{\tilde{t}_{2}}^{2} ; m_{t}, m_{\tilde{g}}\right)\right]
\end{aligned}
$$

where $B_{1}$ is the coefficient of the two-point tensor integral of rank one and $A_{0}$ denotes the scalar one-point function, cf. appendix A. The wave function corrections can be cast into the form

$$
\begin{aligned}
\delta Z_{\tilde{t}_{1} \tilde{t}_{1}}= & \operatorname{Re}\left[-4 m_{t} m_{\tilde{g}} \sin \left(2 \theta_{\tilde{t}}\right) B_{0}^{\prime}\left(m_{\tilde{t}_{1}}^{2} ; m_{t}, m_{\tilde{g}}\right)+2\left(m_{\tilde{g}}^{2}+m_{t}^{2}-m_{\tilde{t}_{1}}^{2}\right) B_{0}^{\prime}\left(m_{\tilde{t}_{1}}^{2} ; m_{t}, m_{\tilde{g}}\right)\right. \\
& \left.+2 B_{0}\left(m_{\tilde{t}_{1}}^{2} ; m_{\tilde{t}_{1}}, \zeta\right)-2 B_{0}\left(m_{\tilde{t}_{1}}^{2} ; m_{t}, m_{\tilde{g}}\right)+4 m_{\tilde{t}_{1}}^{2} B_{0}^{\prime}\left(m_{\tilde{t}_{1}}^{2} ; m_{\tilde{t}_{1}}, \zeta\right)\right]
\end{aligned}
$$




$$
\begin{aligned}
\delta Z_{\tilde{t}_{2} \tilde{t}_{2}}= & \operatorname{Re}\left[4 m_{t} m_{\tilde{g}} \sin \left(2 \theta_{\tilde{t}}\right) B_{0}^{\prime}\left(m_{\tilde{t}_{2}}^{2} ; m_{t}, m_{\tilde{g}}\right)+2\left(m_{\tilde{g}}^{2}+m_{t}^{2}-m_{\tilde{t}_{2}}^{2}\right) B_{0}^{\prime}\left(m_{\tilde{t}_{2}}^{2} ; m_{t}, m_{\tilde{g}}\right)\right. \\
& \left.+2 B_{0}\left(m_{\tilde{t}_{2}}^{2} ; m_{\tilde{t}_{2}}, \zeta\right)-2 B_{0}\left(m_{\tilde{t}_{2}}^{2} ; m_{t}, m_{\tilde{g}}\right)+4 m_{\tilde{t}_{2}}^{2} B_{0}^{\prime}\left(m_{\tilde{t}_{2}}^{2} ; m_{\tilde{t}_{2}}, \zeta\right)\right] .
\end{aligned}
$$

Here $B_{0}^{\prime}\left(k^{2} ; m_{1}, m_{2}\right)$ denotes the derivative with respect to $k^{2}$. The mixing angle counterterm is renormalized as

$$
\delta \theta_{\tilde{t}}=\frac{1}{2}\left(\delta Z_{\tilde{t}_{1} \tilde{t}_{2}}-\delta Z_{\tilde{t}_{2} \tilde{t}_{1}}\right)=\frac{1}{2\left(m_{\tilde{t}_{1}}^{2}-m_{\tilde{t}_{2}}^{2}\right)} \operatorname{Re}\left[\Sigma_{\tilde{t}_{1} \tilde{t}_{2}}\left(m_{\tilde{t}_{2}}^{2}\right)+\Sigma_{\tilde{t}_{2} \tilde{t}_{1}}\left(m_{\tilde{t}_{1}}^{2}\right)\right]
$$

where $\Sigma_{\tilde{t}_{i} \tilde{t}_{j}}$ denotes the respective squark self-energies, so that

$$
\begin{aligned}
\delta \theta_{\tilde{t}}= & \frac{1}{m_{\tilde{t}_{1}}^{2}-m_{\tilde{t}_{2}}^{2}} \operatorname{Re}\left[\sin \left(2 \theta_{\tilde{t}}\right) \cos \left(2 \theta_{\tilde{t}}\right)\left(A_{0}\left(m_{\tilde{t}_{2}}^{2}\right)-A_{0}\left(m_{\tilde{t}_{1}}^{2}\right)\right)\right. \\
& \left.+2 m_{t} m_{\tilde{g}} \cos \left(2 \theta_{\tilde{t}}\right)\left(B_{0}\left(m_{\tilde{t}_{2}}^{2} ; m_{t}, m_{\tilde{g}}\right)+B_{0}\left(m_{\tilde{t}_{1}}^{2} ; m_{t}, m_{\tilde{g}}\right)\right)\right] .
\end{aligned}
$$

The real corrections finally in terms of dilogarithms read

$$
\begin{aligned}
\Delta_{A_{k}}^{R}= & \frac{2 G_{A_{k}}^{12}}{\lambda^{1 / 2}}\left[( M _ { A _ { k } } ^ { 2 } - m _ { \tilde { t } _ { 1 } } ^ { 2 } - m _ { \tilde { t } _ { 2 } } ^ { 2 } ) \left(-2 \log \beta_{0} \log \frac{\zeta M_{A_{k}} m_{\tilde{t}_{1}} m_{\tilde{t}_{2}}}{\lambda}+2 \log ^{2} \beta_{0}\right.\right. \\
& \left.-\log ^{2} \beta_{1}-\log ^{2} \beta_{2}+2 \operatorname{Li}_{2}\left(1-\beta_{0}^{2}\right)-\operatorname{Li}_{2}\left(1-\beta_{1}^{2}\right)-\operatorname{Li}_{2}\left(1-\beta_{2}^{2}\right)\right) \\
& +2 \lambda^{1 / 2} \log \frac{\zeta M_{A_{k}} m_{\tilde{t}_{1}} m_{\tilde{t}_{2}}}{\lambda}+4 \lambda^{1 / 2}+\left(2 M_{A_{k}}^{2}+m_{\tilde{t}_{1}}^{2}+m_{\tilde{t}_{2}}^{2}\right) \log \beta_{0} \\
& \left.+\left(M_{A_{k}}^{2}+2 m_{\tilde{t}_{2}}^{2}\right) \log \beta_{2}+\left(M_{A_{k}}^{2}+2 m_{\tilde{t}_{1}}^{2}\right) \log \beta_{1}\right]
\end{aligned}
$$

where in the two-body phase space function $\lambda\left(M_{A_{k}}^{2}, m_{\tilde{t}_{1}}^{2}, m_{\tilde{t}_{2}}^{2}\right)$ we have neglected the arguments for better readability. We have furthermore introduced

$$
\begin{gathered}
\beta_{0}=\frac{M_{A_{k}}^{2}-m_{\tilde{t}_{1}}^{2}-m_{\tilde{t}_{2}}^{2}+\lambda^{1 / 2}}{2 m_{\tilde{t}_{1}} m_{\tilde{t}_{2}}}, \\
\beta_{1}=\frac{M_{A_{k}}^{2}-m_{\tilde{t}_{1}}^{2}+m_{\tilde{t}_{2}}^{2}-\lambda^{1 / 2}}{2 M_{A_{k}} m_{\tilde{t}_{2}}}, \\
\beta_{2}=\frac{M_{A_{k}}^{2}+m_{\tilde{t}_{1}}^{2}-m_{\tilde{t}_{2}}^{2}-\lambda^{1 / 2}}{2 M_{A_{k}} m_{\tilde{t}_{1}}} .
\end{gathered}
$$

We explicitly checked that the dependence on the fictitious gluon mass drops out in the final result, as it should be.

We have cross-checked our results against the ones available in the literature for the MSSM [83, 84], both on the analytical and on the numerical level. For the former we performed the MSSM limit of our formulae, and for the latter we took the MSSM limit of the input parameters to evaluate the decay widths. 


\section{The one-loop electroweak corrections}

The NLO electroweak corrections consist of the virtual corrections to the vertex and the counterterm contributions to cancel the UV divergences. The top and stop fields as well as the parameters specified below need to be renormalized. For the external Higgs boson we use the two-loop corrected Higgs boson mass at order $\mathcal{O}\left(\alpha_{t} \alpha_{s}\right)$, including the full EW corrections at one-loop order, so that we also need to renormalize the Higgs field. In the loops, however, the tree-level masses for the Higgs bosons have to be used so that the UV divergences are canceled properly.

For the NLO EW corrections, in addition the mixings

$$
\delta M_{\mathrm{mix}, i}^{G, Z} \equiv \delta M_{\mathrm{mix}, i}^{G, Z}\left(A_{i} \rightarrow \tilde{t}_{1} \tilde{t}_{2}\right)
$$

of the decaying CP-odd Higgs boson $A_{i}$ with the $Z$ boson and the Goldstone boson $G$ have to be included. The matrix element for the EW corrected pseudoscalar Higgs decay into stops hence reads

$$
\mathcal{M}\left(A_{i} \rightarrow \tilde{t}_{1} \tilde{t}_{2}\right)=\sum_{j=1}^{2} \mathbf{Z}_{i j}\left(-G_{A_{j}}^{12}+\Delta_{A_{j}}^{\mathrm{EW}}\right)+\delta M_{\text {mix }, i}^{G, Z}
$$

where $\Delta_{A_{j}}^{\mathrm{EW}}$ represents the sum of the 1-particle irreducible (1PI) diagrams $\Delta_{A_{j}}^{V \text {,EW }}$ contributing to the EW virtual corrections of the vertex and of the counterterms $\Delta_{A_{j}}^{\mathrm{CT}, \mathrm{EW}}$,

$$
\Delta_{A_{j}}^{\mathrm{EW}}=\Delta_{A_{j}}^{V, \mathrm{EW}}+\Delta_{A_{j}}^{\mathrm{CT}, \mathrm{EW}}
$$

Due to massless photons in the loops we also encounter IR divergences. These are canceled by adding the real corrections $\Delta^{R, \mathrm{EW}}$, where a photon is radiated off the final state stop lines. We hence have for the EW corrected decay amplitude

$$
\begin{aligned}
\Gamma_{\mathrm{EW}}^{\mathrm{NLO}}\left(A_{i} \rightarrow \tilde{t}_{1} \tilde{t}_{2}\right)=\frac{3 \lambda^{1 / 2}}{8 \pi M_{A_{i}}^{3}} & {\left[\left|\sum_{j=1}^{2} \mathbf{Z}_{i j} G_{A_{j}}^{12}\right|^{2}+\left(\sum_{j=1}^{2} \mathbf{Z}_{i j}^{*} G_{A_{j}}^{12} *\right)\left(\sum_{k=1}^{2} \mathbf{Z}_{i k} \Delta_{A_{k}}^{R, \mathrm{EW}}\right)\right.} \\
& \left.-2 \operatorname{Re}\left(\sum_{j=1}^{2} \mathbf{Z}_{i j}^{*} G_{A_{j}}^{12 *}\left(\sum_{k=1}^{2} \mathbf{Z}_{i k} \Delta_{A_{k}}^{\mathrm{EW}}+\delta M_{\mathrm{mix}, \mathrm{i}}^{G, Z}\right)\right)\right] .
\end{aligned}
$$

Again we have dropped the arguments in the two-body phase space function $\lambda$. In the following we will discuss the individual contributions. The virtual corrections consist of the 1PI diagrams given by the triangle diagrams with scalars, fermions and gauge bosons in the loops, as shown in the first two rows of figure 3 , and of the diagrams involving fourparticle vertices, cf. figure 3 (last row). For better readability, for the scalars $S$ appearing in the loops we only list the particle types but not the combination of scalars that are allowed by the theory for the various vertices. Let us remark, however, that in the fourparticle vertices the scalar-Higgs-Goldstone-boson-2-stops coupling $H_{l}-G-\tilde{t}_{1}-\tilde{t}_{2}$ and the scalar-pseudoscalar-2-stops coupling $H_{l}-A_{p}-\tilde{t}_{1}-\tilde{t}_{2}(l=1,2,3, p=1,2)$ are new 
compared to the MSSM. The former is due to the singlet admixture in $H_{l}$, the latter is proportional to the NMSSM specific coupling $\lambda$. The diagrams have been generated with the Mathematica package FeynArts $3.6[129,130]$ and evaluated with FormCalc 7.3 [131, 132 ] in two independent calculations. The integrals have been computed with LoopTools $2.7[131,132]$. The numerical results of both calculations agree and have been cross-checked numerically against a third calculation, that did not use any of the tools to evaluate and simplify the amplitudes, and which takes the loop functions from HDECAY [133-135] and SDECAY [136, 137]. The UV divergences encountered in the computation of the virtual corrections are canceled by the counterterms that are the sum of the stop wave function corrections and of the counterterm renormalizing the $A_{j} \tilde{t}_{1} \tilde{t}_{2}$ interaction,

$$
\Delta_{A_{j}}^{\mathrm{CT}, \mathrm{EW}}=\Delta_{A_{j}}^{\mathrm{CT}, w}+\Delta_{A_{j}}^{\mathrm{CT}, v}
$$

Because of the antisymmetric $G_{A_{j}}^{12}$ coupling the stop wave function corrections are given by

$$
\Delta_{A_{j}}^{\mathrm{CT}, w}=-\frac{G_{A_{j}}^{12}}{2}\left(\delta Z_{\tilde{t}_{1} \tilde{t}_{1}}+\delta Z_{\tilde{t}_{2} \tilde{t}_{2}}\right) .
$$

The stops are renormalized on-shell, with the renormalization conditions given in eqs. (4.12) and (4.13). The diagrams, that contribute to the here required electroweak self-energies are displayed in figure 4 (upper two rows).

For the one-loop EW corrections the vertex counterterm reads

$$
\begin{aligned}
\Delta_{A_{j}}^{\mathrm{CT}, v}= & -G_{A_{j}}^{12}\left(\frac{\delta g}{g}+\frac{\delta m_{t}}{m_{t}}-\frac{\delta M_{W}}{M_{W}}\right)-\frac{g m_{t}}{2 M_{W}}\left[\mathcal{R}_{j 1}^{P}\left(\delta \mu+\left(\frac{\delta A_{t}}{A_{t}}-\frac{\delta \tan \beta}{\tan \beta}\right) \frac{A_{t}}{\tan \beta}\right)\right. \\
& \left.+\left(\frac{\delta \lambda}{\lambda}+\frac{\delta v}{v}-\frac{\delta \tan \beta}{\tan \beta}\right) \frac{\lambda v}{\sqrt{2} \tan \beta} \mathcal{R}_{j 2}^{P}\right] .
\end{aligned}
$$

The individual counterterms are derived from the renormalization of the input parameters. We follow ref. [103] and apply the same renormalization scheme which mixes OS and $\overline{\mathrm{DR}}$ conditions as defined there. For the vertex counterterms the relevant input parameters are the $W$ and $Z$ boson masses $M_{W}$ and $M_{Z}$, the electric charge $e, \tan \beta, \lambda$ and $v_{s} .{ }^{3}$ The parameters that can be related to physical quantities are renormalized OS, the remaining ones $\overline{\mathrm{DR}}$. Together with the OS-renormalized top/stop sector, we have the following set of parameters to be renormalized,

$$
\underbrace{\tan \beta, \lambda, v_{s}}_{\overline{\mathrm{DR}} \text { scheme }}, \underbrace{M_{Z}, M_{W}, e, m_{t}, m_{\tilde{t}_{1}}, m_{\tilde{t}_{2}}, \theta_{\tilde{t}}}_{\text {OS scheme }} .
$$

The coupling $g$ and the VEV $v$ appearing in eq. (5.7) are given in terms of these parameters by

$$
g=\frac{e M_{Z}}{\sqrt{M_{Z}^{2}-M_{W}^{2}}} \quad \text { and } \quad v=\frac{2 M_{W}}{e} \sqrt{1-\frac{M_{W}^{2}}{M_{Z}^{2}}}
$$

\footnotetext{
${ }^{3}$ Additionally, for the renormalization of the Higgs sector in the computation of the higher order corrections to the Higgs boson masses, we have the tadpole parameters, the mass of the charged Higgs boson $M_{H^{ \pm}}$, the NMSSM parameter $\kappa$ and the trilinear coupling $A_{\kappa}$, which need to be renormalized at loop level, cf. [103].
} 


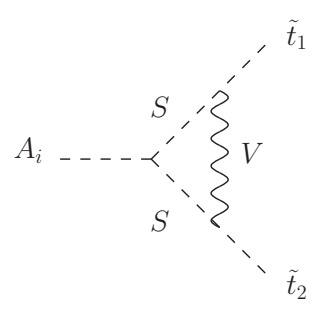

$(S, V)=\left(\tilde{t}_{j} / \tilde{t}_{k}, \gamma / Z\right),\left(\tilde{b}_{j} / \tilde{b}_{k}, W^{ \pm}\right)$

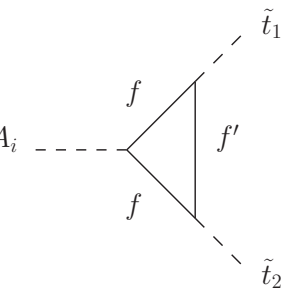

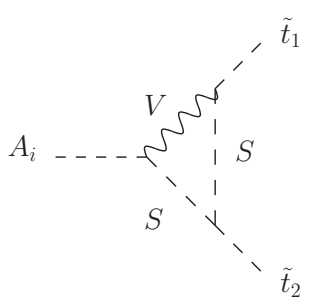

$(S, V)=\left(\tilde{t}_{j} / \tilde{t}_{k} / H_{l}, Z\right),\left(\tilde{b}_{j} / \tilde{b}_{k} / H^{ \pm} / G^{ \pm}, W^{ \pm}\right)$

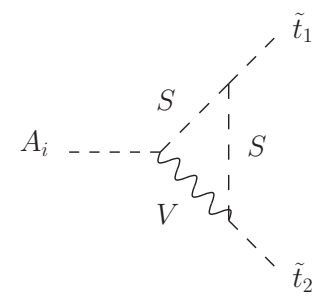

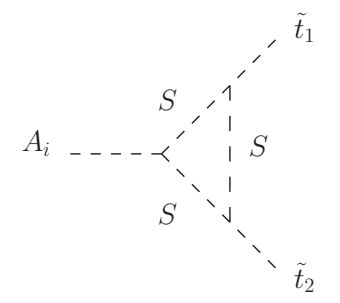

$\left(f, f^{\prime}\right)=\left(t / b, \tilde{\chi}_{m}^{0} / \tilde{\chi}_{j}^{ \pm}\right),\left(\tilde{\chi}_{m}^{0} / \tilde{\chi}_{n}^{0}, t\right),\left(\tilde{\chi}_{j}^{ \pm} / \tilde{\chi}_{k}^{ \pm}, b\right)$

$S=\tilde{t}_{j}, \tilde{b}_{k}, H_{l}, A_{p}, G^{0}, H^{ \pm}, G^{ \pm}$

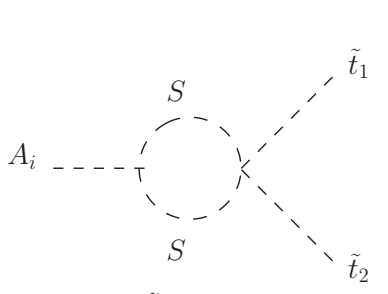

$S=\tilde{q}_{i}, \tilde{l}_{k}, \tilde{\nu}, H_{l}, A_{p}, G^{0}$

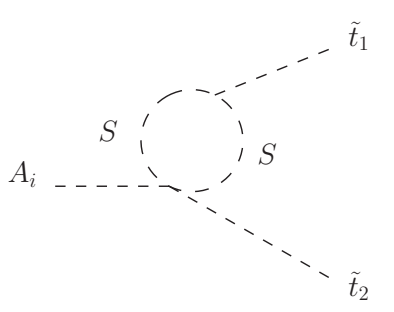

$S=\tilde{t}_{j}, \tilde{b}_{k}, A_{p}, G^{0}, H^{ \pm}, G^{ \pm}$

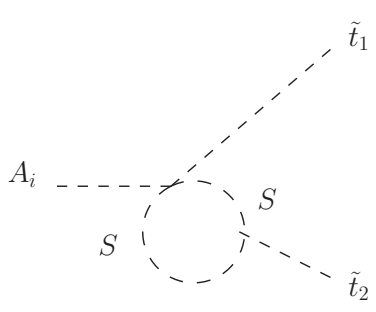

$H^{ \pm}, G^{ \pm}$

Figure 3. Generic diagrams contributing to the electroweak corrections of the decay $\Gamma\left(A_{i} \rightarrow \tilde{t}_{1} \tilde{t}_{2}\right)$ with $i, j, k, p=1,2, l=1,2,3$ and $m, n=1, \ldots, 5$.
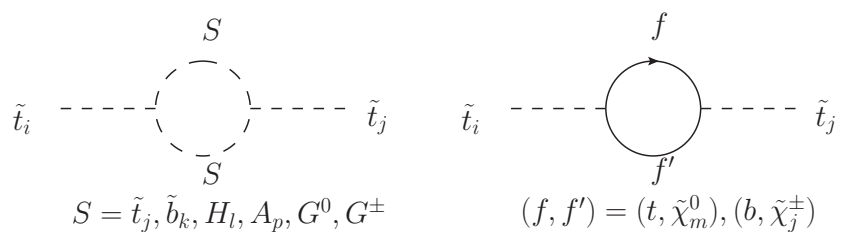

$\left(f, f^{\prime}\right)=\left(t, \tilde{\chi}_{m}^{0}\right),\left(b, \tilde{\chi}_{j}^{ \pm}\right)$

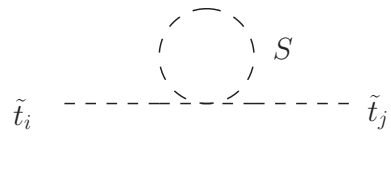

$S=\tilde{q}_{j}, \tilde{l}_{k}, \tilde{\nu}, H_{l}, A_{p}, H^{ \pm}, G^{0}, G^{ \pm}$

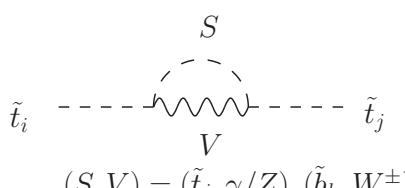

$(S, V)=\left(\tilde{t}_{j}, \gamma / Z\right),\left(\tilde{b}_{k}, W^{ \pm}\right)$

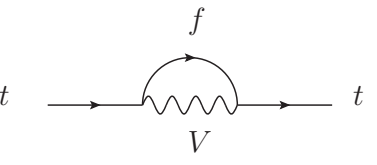

$(f, V)=(t, \gamma / Z),\left(b, W^{ \pm}\right)$

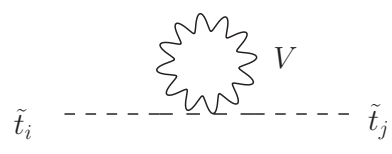

$V=\gamma, Z, W^{ \pm}$

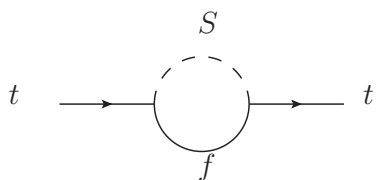

$(S, f)=\left(H_{l} / G^{0}, t\right),\left(H^{ \pm} / G^{ \pm}, b\right),\left(\tilde{t}_{j}, \tilde{\chi}_{m}^{0}\right),\left(\tilde{b}_{k}, \tilde{\chi}_{p}^{ \pm}\right)$

Figure 4. Diagrams contributing to the electroweak squark self-energies (first two rows) and quark self-energies (last row) with $i, j, k, p=1,2, l=1,2,3$ and $m=1, \ldots, 5$. 

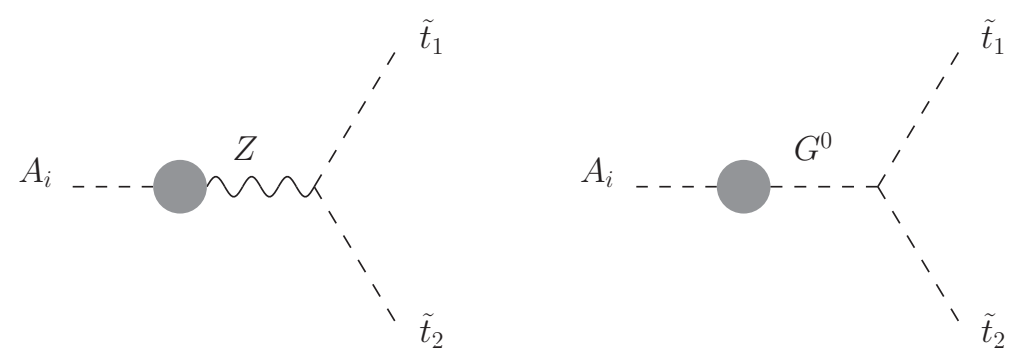

Figure 5. Generic one-loop diagrams contributing to the mixing $\delta M_{\mathrm{mix}, i}^{G, Z}$ of a pseudoscalar $A_{i}$ with the $Z$ and Goldstone boson.

from which their counterterms can be derived. The details of the renormalization of the counterterms for the first six input parameters can be found in [103], so that they are not repeated here. The formulae for the OS renormalization of the top and stop masses are given in eqs. (4.11)-(4.13). The squark and quark self-energies for the EW one-loop corrections are depicted in figure 4. At EW one-loop order the counterterm for $A_{t}$ now reads

$$
\begin{aligned}
\delta A_{t}= & \frac{\mu}{\tan \beta}\left(\frac{\delta \mu}{\mu}-\frac{\delta \tan \beta}{\tan \beta}\right)+\frac{1}{2 m_{t}}\left[\left(m_{\tilde{t}_{1}}^{2}-m_{\tilde{t}_{2}}^{2}\right)\left(2 \delta \theta_{\tilde{t}} \cos \left(2 \theta_{\tilde{t}}\right)-\sin \left(2 \theta_{\tilde{t}}\right) \frac{\delta m_{t}}{m_{t}}\right)\right. \\
& \left.+2 \sin \left(2 \theta_{\tilde{t}}\right)\left(m_{\tilde{t}_{1}} \delta m_{\tilde{t}_{1}}-m_{\tilde{t}_{2}} \delta m_{\tilde{t}_{2}}\right)\right] .
\end{aligned}
$$

The counterterm for the mixing angle $\theta_{\tilde{t}}$ is renormalized as in eq. (4.19), however with the self-energies given by the diagrams shown in figure 4 (first two rows).

The diagrams for the contributions to the electroweak corrections stemming from the mixings of the pseudoscalar $A_{i}$ with the $Z$ boson and the Goldstone boson, $\delta M_{\mathrm{mix}, i}^{G, Z}$, are shown in figure 5. Using the Slavnov-Taylor identity [138, 139], on can obtain the mixing contributions through

$$
\delta M_{\mathrm{mix}, i}^{G, Z}=\frac{G_{G}^{12}}{M_{Z}} \hat{\Sigma}_{A_{i} Z}\left(\left(M_{A_{i}}^{(0)}\right)^{2}\right)
$$

where $\hat{\Sigma}_{A_{i} Z}$ denotes the renormalized $A_{i}$ - $Z$ mixing self-energy and $G_{G}^{12}$ is the Goldstone boson coupling to the stops,

$$
G_{G}^{12}=-\frac{g m_{t}}{2 M_{W}}\left(A_{t}-\frac{\mu}{\tan \beta}\right) .
$$

Note, in particular that the external momenta have to be set to the tree-level mass $M_{A_{i}}^{(0)}$ in order to ensure gauge invariance.

The last piece which is missing in the decay, in order to get also an IR finite result, is the real corrections term $\Delta_{A_{k}}^{R, \mathrm{EW}}$. This is the same as for the QCD corrections, but with the gluon replaced by the photon. In the formula for the QCD corrections, eq. (4.21), this means that the coupling and color factors have to be replaced accordingly, i.e.

$$
\Delta_{A_{k}}^{R, \mathrm{EW}}=\frac{e^{2}}{16 \pi^{2}} \Delta_{A_{k}}^{R}
$$

with $\Delta_{A_{k}}^{R}$ given in eq. (4.21). 
The full NLO decay width including the SUSY-QCD and -EW corrections is then given by

$$
\Gamma^{\mathrm{NLO}, \text { full }}=\Gamma_{\mathrm{EW}}^{\mathrm{NLO}}+\Gamma_{\mathrm{QCD}}^{(1)}
$$

where $\Gamma_{\mathrm{EW}}^{\mathrm{NLO}}$, defined in eq. (5.4), includes the leading order decay width and $\Gamma_{\mathrm{QCD}}^{(1)}$ has been defined in eq. (4.2).

\section{Stop decays at NLO SUSY-QCD and SUSY-EW}

The calculation of the higher order corrections to the pseudoscalar Higgs decays into a stop pair can easily be transferred to the decay of a heavy stop into a pseudoscalar and the light stop,

$$
\tilde{t}_{2} \rightarrow A_{i}+\tilde{t}_{1} \quad i=1,2 .
$$

The LO decay width is obtained from eq. (3.10) by adapting the kinematic factor and dividing by the color factor 3 and the factor 2 due to the summation of the two charge conjugated stop pair final states,

$$
\Gamma^{\mathrm{LO}}\left(\tilde{t}_{2} \rightarrow A_{i} \tilde{t}_{1}\right)=\frac{\lambda^{1 / 2}\left(m_{\tilde{t}_{2}}^{2}, M_{A_{i}}^{2}, m_{\tilde{t}_{1}}^{2}\right)}{16 \pi m_{\tilde{t}_{2}}^{3}}\left|\sum_{j=1}^{2} \mathbf{Z}_{i j} G_{A_{j}}^{12}\right|^{2}
$$

The NLO SUSY-QCD decay width can be written as

$$
\Gamma^{\mathrm{NLO}}\left(\tilde{t}_{2} \rightarrow A_{i} \tilde{t}_{1}\right)=\Gamma^{\mathrm{LO}}\left(\tilde{t}_{2} \rightarrow A_{i} \tilde{t}_{1}\right)+\Gamma_{\mathrm{QCD}}^{(1)}\left(\tilde{t}_{2} \rightarrow A_{i} \tilde{t}_{1}\right)
$$

where

$$
\Gamma_{\mathrm{QCD}}^{(1)}\left(\tilde{t}_{2} \rightarrow A_{i} \tilde{t}_{1}\right)=\operatorname{Re}\left[\frac{\lambda^{1 / 2}\left(m_{\tilde{t}_{2}}^{2}, M_{A_{i}}^{2}, m_{\tilde{t}_{1}}^{2}\right)}{24 \pi m_{\tilde{t}_{2}}^{3}}\left(\sum_{j=1}^{2} \mathbf{Z}_{i j}^{*} G_{A_{j}}^{12}\right) \frac{\alpha_{s}}{\pi}\left(\sum_{k=1}^{2} \mathbf{Z}_{i k} \Delta_{k, \tilde{t}_{2}}^{\mathrm{QCD}}\right)\right]
$$

with the correction factor

$$
\Delta_{k, \tilde{t}_{2}}^{\mathrm{QCD}}=\Delta_{k, \tilde{t}_{2}}^{V}+\Delta_{k, \tilde{t}_{2}}^{\mathrm{CT}}+\Delta_{k, \tilde{t}_{2}}^{R}
$$

The virtual corrections ${ }^{4}$ and the counterterms are given by the same expressions as for the pseudoscalar decay presented in section 4 , i.e.

$$
\Delta_{k, \tilde{t}_{2}}^{V}=\Delta_{A_{k}}^{V} \quad \text { and } \quad \Delta_{k, \tilde{t}_{2}}^{\mathrm{CT}}=\Delta_{A_{k}}^{\mathrm{CT}} .
$$

In the real corrections, though, the roles of $A_{k}$ and $\tilde{t}_{2}$ have to be interchanged,

$$
\Delta_{k, \tilde{t}_{2}}^{R}=\Delta_{A_{k}}^{R}\left(M_{A_{k}}^{2} \leftrightarrow m_{\tilde{t}_{2}}^{2}\right)
$$

\footnotetext{
${ }^{4}$ The diagrams are the same as in figure 1 , but with $\tilde{t}_{2}$ in the initial and $A_{i}$ in the final state.
} 
with $\Delta_{A_{k}}^{R}$ given in eq. (4.21). The NLO SUSY-EW corrections are composed of the same electroweak virtual corrections to the vertex,${ }^{5}$ the same counterterms and the same mixing contributions of the pseudoscalar with the $Z$ and Goldstone boson, $\Delta_{A_{k}}^{\mathrm{EW}}$ and $\delta M_{\mathrm{mix}, i}^{G, Z}$, as in the pseudoscalar decay. In the real corrections, however, again $M_{A_{i}}^{2}$ and $m_{\tilde{t}_{2}}^{2}$ have to be interchanged. We hence have

$$
\begin{aligned}
\Gamma_{\mathrm{EW}}^{\mathrm{NLO}}\left(\tilde{t}_{2} \rightarrow A_{i} \tilde{t}_{1}\right)=\frac{\lambda^{1 / 2}}{16 \pi m_{\tilde{t}_{2}}^{3}} & {\left[\left|\sum_{j=1}^{2} \mathbf{Z}_{i j} G_{A_{j}}^{12}\right|^{2}+\left(\sum_{j=1}^{2} \mathbf{Z}_{i j}^{*} G_{A_{j}}^{12 *}\right)\left(\sum_{k=1}^{2} \mathbf{Z}_{i k} \Delta_{k, \tilde{t}_{2}}^{R, \mathrm{EW}}\right)\right.} \\
& \left.-2 \operatorname{Re}\left(\sum_{j=1}^{2} \mathbf{Z}_{i j}^{*} G_{A_{j}}^{12 *}\left(\sum_{k=1}^{2} \mathbf{Z}_{i k} \Delta_{A_{k}}^{\mathrm{EW}}+\delta M_{\mathrm{mix}, i}^{G, Z}\right)\right)\right],
\end{aligned}
$$

with

$$
\lambda^{1 / 2} \equiv \lambda^{1 / 2}\left(m_{\tilde{t}_{2}}^{2}, M_{A_{i}}^{2}, m_{\tilde{t}_{1}}^{2}\right)
$$

and

$$
\Delta_{k, \tilde{t}_{2}}^{R, \mathrm{EW}}=\frac{e^{2}}{16 \pi^{2}} \Delta_{A_{k}}^{R}\left(M_{A_{k}}^{2} \leftrightarrow m_{\tilde{t}_{2}}^{2}\right)
$$

and $\Delta_{A_{k}}^{\mathrm{EW}}$ and $\delta M_{\mathrm{mix}, i}^{G, Z}$ given in section 5. The full NLO decay width including SUSY-QCD and -EW corrections is given by

$$
\Gamma^{\mathrm{NLO}, \text { full }}=\Gamma_{\mathrm{EW}}^{\mathrm{NLO}}+\Gamma_{\mathrm{QCD}}^{(1)}
$$

with $\Gamma_{\mathrm{QCD}}^{(1)}$ given by eqs. (6.4)-(6.7) and $\Gamma_{\mathrm{EW}}^{\mathrm{NLO}}$ by eq. (6.8).

\section{Numerical analysis}

For our numerical analysis we first perform a scan in the NMSSM parameter space in order to find scenarios that are in accordance with the LHC Higgs and SUSY data. The compatibility with the LHC Higgs data has been checked by using the programs HiggsBounds [140142] and HiggsSignals [143]. The effective couplings of the NMSSM Higgs bosons, normalized to the corresponding SM values, as well as the masses, the widths and the branching ratios of the NMSSM Higgs bosons, which are required as inputs for these programs, have been obtained from the Fortran code NMSSMCALC [120]. The loop induced Higgs coupling to gluons normalized to the corresponding coupling of a SM Higgs boson with same mass is obtained by taking the ratio of the partial widths for the Higgs decays into gluons in the NMSSM and the SM, respectively. These include the QCD corrections up to next-to-nextto-next-to leading order in the limit of heavy quarks [144-153] and squarks [154, 155], taken over from the SM, respectively, MSSM case, while the EW corrections are unknown for the SUSY case and hence consistently neglected also in the SM decay width. The stop mass

\footnotetext{
${ }^{5}$ In the Feynman diagrams of figure 3 simply the $\tilde{t}_{2}$ leg has to be crossed to the initial state and the $A_{i}$ leg to the final state.
} 
values have been chosen such that they are not excluded by present ATLAS [156-163] and CMS [164-170] searches. The squark masses of the first two generations are heavy enough not to be in conflict with LHC data. The SM input parameters that we use are $[171,172]$

$$
\begin{aligned}
& \alpha\left(M_{Z}\right)=1 / 128.962, \quad \alpha_{s}^{\overline{\mathrm{MS}}}\left(M_{Z}\right)=0.1184, \quad M_{Z}=91.1876 \mathrm{GeV}, \\
& M_{W}=80.385 \mathrm{GeV}, \quad m_{t}=173.5 \mathrm{GeV}, \quad m_{b}^{\overline{\mathrm{MS}}}\left(m_{b}^{\overline{\mathrm{MS}}}\right)=4.19 \mathrm{GeV} .
\end{aligned}
$$

The running $\alpha_{s}^{\overline{\mathrm{DR}}}$ used in NMSSMCALC is obtained by converting the $\alpha_{s}^{\overline{\mathrm{MS}}}$, that is evaluated with the SM renormalization group equations at two-loop order, to the $\overline{\mathrm{DR}}$ scheme. The light quark masses, which have only a small influence on the loop results, have been set to

$$
m_{u}=2.5 \mathrm{MeV}, \quad m_{d}=4.95 \mathrm{MeV}, \quad m_{s}=100 \mathrm{MeV} \text { and } m_{c}=1.42 \mathrm{GeV} .
$$

We choose the same renormalization scale $\mu_{R}$ as the one used in the calculation of the loop-corrected Higgs boson masses, that enter the decay widths. It is given by the SUSY scale $M_{s}$, which we set

$$
M_{s}=\sqrt{m_{\tilde{Q}_{3}} m_{\tilde{t}_{R}}}=\mu_{R} .
$$

\subsection{Pseudoscalar Higgs boson decays into stop pairs}

In this subsection we present the impact of the SUSY-QCD and -EW corrections on the decay of a heavy pseudoscalar Higgs boson into a pair of stop quarks. We will investigate the corrections as a function of $m_{\tilde{t}_{1}}$ and $m_{\tilde{g}}$. They have the largest impact on the genuine corrections to the decay widths. The impact of the other parameters is either tiny or they enter the Higgs sector already at tree level with a large impact on the pseudoscalar mass value, so that the genuine effect of the higher order corrections on the decay widths cannot be disentangled any more. The parameter point, which we have chosen from the set of parameter points that survive the LHC constraints, is given by the soft SUSY breaking masses and trilinear couplings

$$
\begin{aligned}
& m_{\tilde{u}_{R}, \tilde{c}_{R}}=m_{\tilde{d}_{R}, \tilde{s}_{R}}=m_{\tilde{Q}_{1,2}}=m_{\tilde{L}_{1,2}}=m_{\tilde{e}_{R}, \tilde{\mu}_{R}}=3 \mathrm{TeV}, m_{\tilde{t}_{R}}=536.43 \mathrm{GeV}, \\
& m_{\tilde{Q}_{3}}=594.61 \mathrm{GeV}, m_{\tilde{b}_{R}}=1285 \mathrm{GeV}, \quad m_{\tilde{L}_{3}}=255.53 \mathrm{GeV}, \quad m_{\tilde{\tau}_{R}}=1499 \mathrm{GeV}, \\
& A_{t}=1418 \mathrm{GeV}, \quad A_{u, c}=1435 \mathrm{GeV}, \quad A_{d, s, b}=-66.68 \mathrm{GeV}, A_{e, \mu, \tau}=-91.76 \mathrm{GeV}, \\
& M_{1}=111.73 \mathrm{GeV}, \quad M_{2}=395.86 \mathrm{GeV}, \quad M_{3}=1370 \mathrm{GeV}
\end{aligned}
$$

and NMSSM specific input parameters

$$
\begin{aligned}
\lambda & =0.629, \quad \kappa=0.223, \quad A_{\kappa}=-543.53 \mathrm{GeV}, \quad \mu_{\mathrm{eff}}=452.61 \mathrm{GeV}, \\
\tan \beta & =1.969, \quad M_{H^{ \pm}}=1024 \mathrm{GeV} .
\end{aligned}
$$

This results in the two-loop corrected mass $M_{A_{2}}$ of the heavy pseudoscalar $A_{2}$ and the stop masses $m_{\tilde{t}_{1,2}}$,

$$
M_{A_{2}}=1012 \mathrm{GeV} \quad m_{\tilde{t}_{1}}=280.78 \mathrm{GeV} \quad \text { and } \quad m_{\tilde{t}_{2}}=709.07 \mathrm{GeV} .
$$



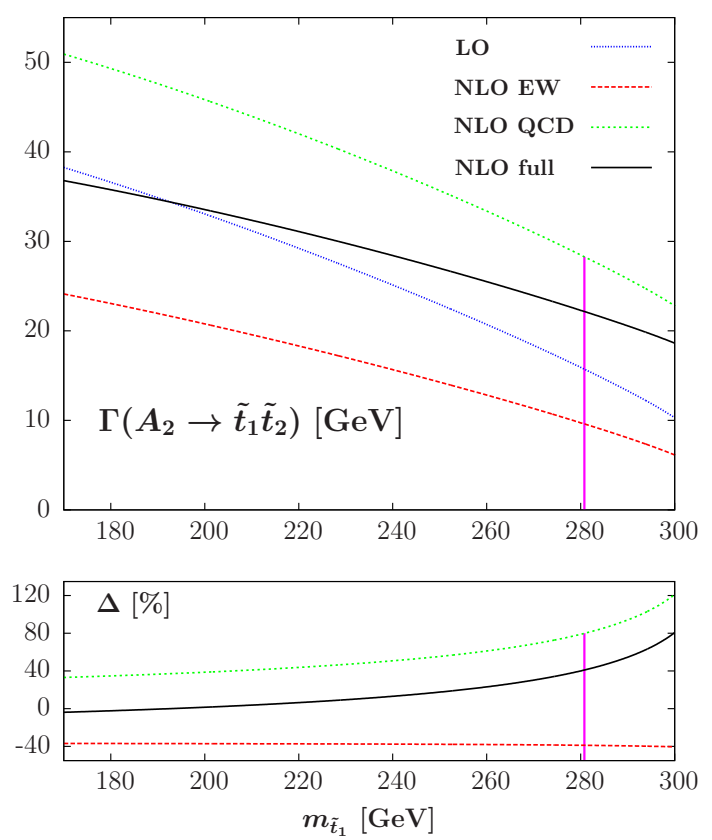

Figure 6. Upper: the partial decay width $\Gamma\left(A_{2} \rightarrow \tilde{t}_{1} \tilde{t}_{2}\right)$ as a function of $m_{\tilde{t}_{1}}$ at LO (blue/lower dotted), including the NLO QCD (green/upper dotted), the NLO EW (red/dashed) and both the EW and QCD corrections (black/full). Lower: the relative correction $\Delta=\left(\Gamma_{X}^{\mathrm{NLO}}-\Gamma^{\mathrm{LO}}\right) / \Gamma^{\mathrm{LO}}$ in per cent for $X=\mathrm{QCD}$ (green/dotted), EW (red/dashed) and the full NLO corrections (black/full). The pink line shows the position of the parameter point defined in eqs. (7.1)-(7.5).

We follow the SLHA format $[173,174]$, in which the parameters $\lambda, \kappa, A_{\kappa}, \mu_{\text {eff }}, \tan \beta$ as well as the soft SUSY breaking masses and trilinear couplings are understood as $\overline{\mathrm{DR}}$ parameters at the scale $\mu_{R}=M_{s}{ }^{6}{ }^{6}$ whereas the charged Higgs mass is an OS parameter. As input for our computation we take the soft SUSY breaking trilinear stop coupling $A_{t}$, however, consistently as an OS parameter. The conversion from the $\overline{\mathrm{DR}}$ to the OS scheme is done within NMSSMCALC and yields ${ }^{7}$

$$
A_{t}^{\mathrm{OS}}=1435 \mathrm{GeV} .
$$

The leading order width obtained in this scenario amounts to

$$
\Gamma^{\mathrm{LO}}\left(A_{2} \rightarrow \tilde{t}_{1} \tilde{t}_{2}\right)=15.72 \mathrm{GeV}
$$

where again we have summed over both charge conjugated stop pair final states. The LO width differs by $2.7 \%$ from the value obtained at tree level with NMSSMCALC, where the Fermi constant $G_{F}$ instead of $\alpha$ is used as input parameter.

In figure 6 (upper) we show the partial decay width $\Gamma\left(A_{2} \rightarrow \tilde{t}_{1} \tilde{t}_{2}\right)$ at LO, including the EW and the QCD corrections, and the NLO width with both the QCD and EW

\footnotetext{
${ }^{6}$ For $\tan \beta$ this is the case only, if it is read in from the block EXTPAR. Otherwise it is the $\overline{\mathrm{DR}}$ parameter at the scale $M_{Z}$.

${ }^{7}$ Note, however, that the conversion is done through a counterterm that involves, as required for the order $\mathcal{O}\left(\alpha_{t} \alpha_{s}\right)$ corrections computed in NMSSMCALC, order $\mathcal{O}\left(\alpha_{s}\right)$ corrections and no EW corrections.
} 
corrections taken into account, as a function of $m_{\tilde{t}_{1}}$, which is varied around the parameter point defined in eqs. (7.1)-(7.5) with $m_{\tilde{t}_{1}} \approx 281 \mathrm{GeV} .{ }^{8}$ Note, that stops can still be rather light $[162,163,170,175,176]$, down to about $240 \mathrm{GeV}$ for arbitrary neutralino masses [163] and even lower when taking into account the actual $\tilde{t}_{1}$ branching ratio [176]. The figure illustrates the effect of the higher order corrections, although the thus obtained parameter configurations are not all in accordance with the applied constraints anymore. The lower plot displays the relative corrections

$$
\Delta=\frac{\Gamma_{X}^{\mathrm{NLO}}-\Gamma^{\mathrm{LO}}}{\Gamma^{\mathrm{LO}}} \quad X=\mathrm{QCD}, \mathrm{EW}, \mathrm{QCD}+\mathrm{EW}
$$

in per cent. The plots show that both the QCD and the EW corrections are significant and come with opposite sign. The QCD corrections increase the LO width by $\sim 40-120 \%$ in the investigated range, depending on the value of $m_{\tilde{t}_{1}}$, whereas the EW corrections are almost independent of $m_{\tilde{t}_{1}}$ and decrease the cross section by $40 \%$. At $m_{\tilde{t}_{1}}=192 \mathrm{GeV}$ the QCD and EW corrections are of same size and cancel each other. The full corrections hence increase the width between $\sim 0-80 \%$, cf. figure 6 (lower). And for our parameter point the total correction is

$$
\Delta \Gamma^{\mathrm{QCD}+\mathrm{EW}}\left(A_{2} \rightarrow \tilde{t}_{1} \tilde{t}_{2}\right)=41 \% .
$$

This plot demonstrates that both the inclusion of the EW and the QCD corrections is required in order to properly predict the decay width.

In figure 7 we show the branching ratios corresponding to the widths of figure 6 . They have been obtained by replacing in NMSSMCALC the corresponding width with our loop corrected width. ${ }^{9}$ The higher order corrections to the remaining decay modes needed in the computation of the total width for the branching ratio, are the ones as implemented in NMSSMCALC. In particular they include the dominant higher order QCD corrections. The decays into a bottom quark pair furthermore take into account the higher order SUSYQCD and the approximate SUSY-EW corrections up to one-loop accuracy. The decays into a strange quark pair include the dominant resummed SUSY-QCD corrections and the ones into a $\tau$ pair the dominant resummed SUSY-EW corrections. For details, we refer the reader to ref. [120] and the references therein. The branching ratio at LO of our investigated parameter point amounts to

$$
\mathrm{BR}^{\mathrm{LO}}\left(A_{2} \rightarrow \tilde{t}_{1} \tilde{t}_{2}\right)=40.8 \%
$$

\footnotetext{
${ }^{8}$ The variation of $m_{\tilde{t}_{1}}$ between 170 and $300 \mathrm{GeV}$ corresponds to a variation of $A_{t}^{\text {OS }}$ between 1371 and $1721 \mathrm{GeV}$.

${ }^{9}$ In NMSSMCALC the SUSY QCD corrections to the decays into squarks, as derived from [86], are taken into account. These include improvements in the decays into sbottoms, which are required in parts of the parameter space, that are not relevant for us. Furthermore, we include the EW corrections. We therefore consistently turned off the corrections implemented in NMSSMCALC in the decays into squarks and included instead our corrections. The formulae for the NLO SUSY-QCD and SUSY-EW corrections to the decays of CP-odd NMSSM Higgs bosons into stops shall be included in NMSSMCALC and will be made publicly available in a future release of the program.
} 

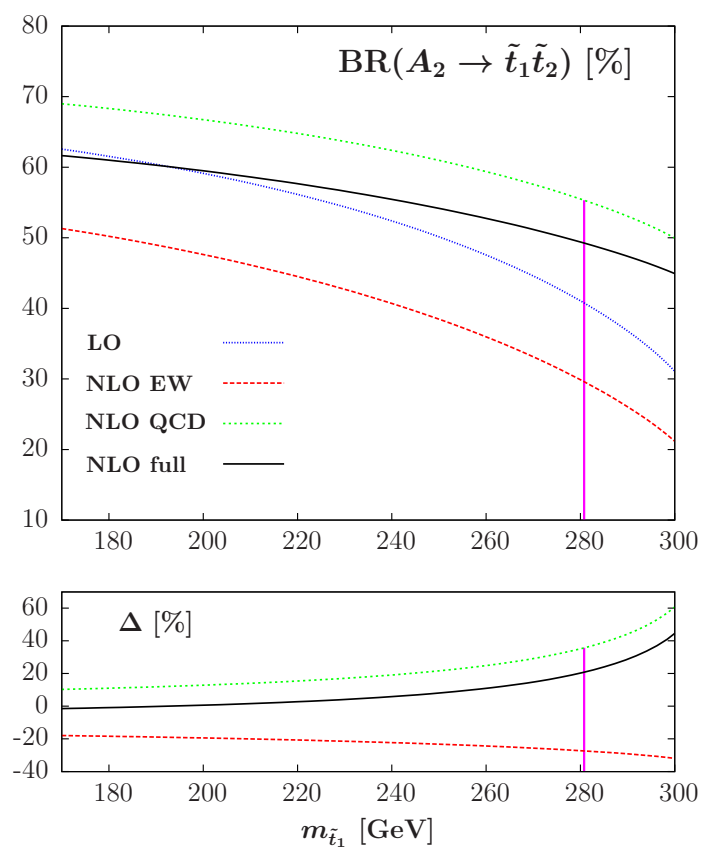

Figure 7. Same as figure 6, but for the branching ratios.

The net effect of the NLO EW and QCD corrections is an increase of the branching ratio by

$$
\Delta \mathrm{BR}^{\mathrm{QCD}+\mathrm{EW}}\left(A_{2} \rightarrow \tilde{t}_{1} \tilde{t}_{2}\right)=+20.8 \% .
$$

In the plot of figure 7 we again vary $m_{\tilde{t}_{1}}$ around the chosen parameter point, illustrated by the pink line in the plot. As can be read off the lower plot the total NLO corrections increase the branching ratio by up to a bit more than $40 \%$ in the shown $m_{\tilde{t}_{1}}$ range. Thus this decay remains the most important one also after the inclusion of the NLO corrections.

Figure 8 finally shows the dependence of the higher order corrections on the gluino mass. The EW corrections of course do not depend on $m_{\tilde{g}}$, while the QCD corrections show a very mild dependence on the gluino mass, apart from the region around $m_{\tilde{g}} \approx$ $535 \mathrm{GeV}$. The kink that appears here, arises in the $\tilde{t}_{2}$ self-energy at the threshold where $m_{\tilde{t}_{2}}=m_{\tilde{g}}+m_{t}$.

The size of the higher order corrections sensitively depends on the scenario. Thus we find for the scenario defined by

$$
\begin{aligned}
& m_{\tilde{u}_{R}, \tilde{c}_{R}}=m_{\tilde{d}_{R}, \tilde{s}_{R}}=m_{\tilde{Q}_{1,2}}=m_{\tilde{L}_{1,2}}=m_{\tilde{e}_{R}, \tilde{\mu}_{R}}=3 \mathrm{TeV}, m_{\tilde{t}_{R}}=714.25 \mathrm{GeV}, \\
& m_{\tilde{Q}_{3}}=1035 \mathrm{GeV}, \quad m_{\tilde{b}_{R}}=2776 \mathrm{GeV}, \quad m_{\tilde{L}_{3}}=2156 \mathrm{GeV}, \quad m_{\tilde{\tau}_{R}}=1755 \mathrm{GeV}, A_{t}=1246 \mathrm{GeV}, \quad A_{u, c}=1347 \mathrm{GeV}, \quad A_{d, s, b}=-1651 \mathrm{GeV}, A_{e, \mu, \tau}=769.08 \mathrm{GeV}, \\
& M_{1}=460.61 \mathrm{GeV}, \quad M_{2}=381.55 \mathrm{GeV}, \quad M_{3}=2296 \mathrm{GeV}
\end{aligned}
$$

and

$$
\begin{aligned}
\lambda & =0.552, \quad \kappa=0.030, \quad A_{\kappa}=-173.51 \mathrm{GeV}, \quad \mu_{\mathrm{eff}}=446.80 \mathrm{GeV}, \\
\tan \beta & =3.005, \quad M_{H^{ \pm}}=1460 \mathrm{GeV},
\end{aligned}
$$



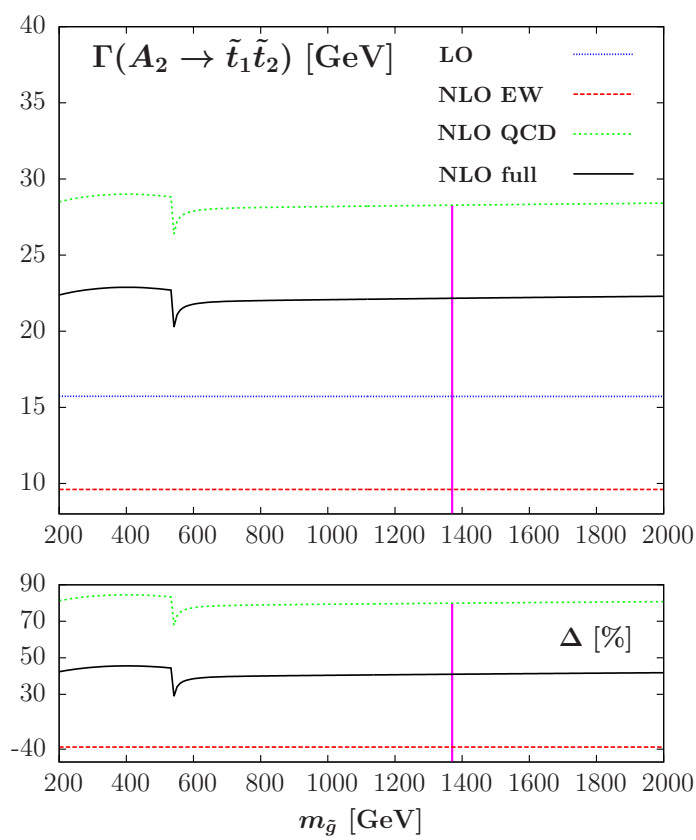

Figure 8. Upper: the partial decay width $\Gamma\left(A_{2} \rightarrow \tilde{t}_{1} \tilde{t}_{2}\right)$ as a function of $m_{\tilde{g}}$. The color and line style code is the same as in figure 6 . Lower: the relative correction $\Delta=\left(\Gamma_{X}^{\mathrm{NLO}}-\Gamma^{\mathrm{LO}}\right) / \Gamma^{\mathrm{LO}}$ in per cent for $X=\mathrm{QCD}$ (green/dotted), EW (red/dashed) and the full NLO corrections (black/full). The pink line shows the position of the parameter point defined in eqs. (7.1)-(7.5).

resulting in

$$
M_{A_{2}}=1461 \mathrm{GeV} \quad m_{\tilde{t}_{1}}=353.02 \mathrm{GeV} \quad m_{\tilde{t}_{2}}=927.56 \mathrm{GeV}
$$

and an LO decay width of

$$
\Gamma^{\mathrm{LO}}\left(A_{2} \rightarrow \tilde{t}_{1} \tilde{t}_{2}\right)=15.24 \mathrm{GeV}
$$

NLO QCD and EW corrections that amount to $\Delta^{\mathrm{QCD}}=23.4 \%$ and $\Delta^{\mathrm{EW}}=-10.2 \%$, respectively, resulting in a total correction of

$$
\Delta^{\mathrm{QCD}+\mathrm{EW}}\left(A_{2} \rightarrow \tilde{t}_{1} \tilde{t}_{2}\right)=13.2 \% .
$$

This can also be inferred from figure 9 which shows the NLO corrections to the decay widths as a function of $m_{\tilde{t}_{1}}$. As demonstrated in the lower plot, the NLO QCD corrections are of the order of $20-25 \%$, while the EW corrections range between about $-17 \%$ and $-8 \%$, leading to an overall increase of the cross section between $3 \%$ and $17 \%$ due to the combined NLO corrections.

\subsection{Stop decays into a pseudoscalar}

Decays of the heavy stop into a pseudoscalar and the light stop can occur and become important when there is a large mass splitting between the two stop mass eigenstates. Stop decays into a SM-like Higgs boson final state have recently been discussed in [177] 

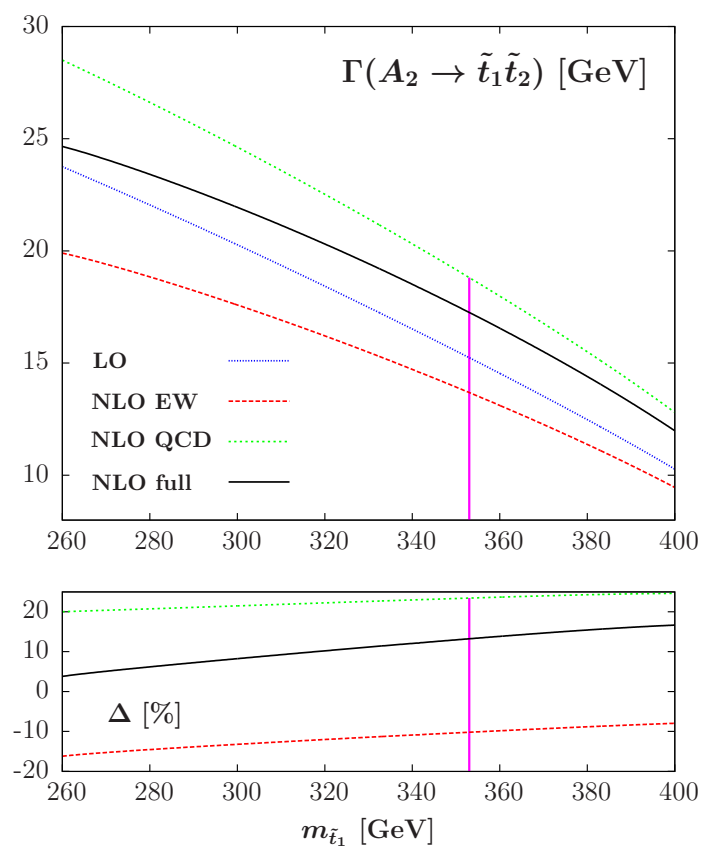

Figure 9. Same as figure 6, but now for the initial scenario (marked by the pink line in the plot) given by eqs. (7.13)-(7.14).

and the production of NMSSM Higgs bosons in squark cascade decays in [76, 178]. In order to show the impact of the SUSY-EW and -QCD corrections on the stop decay width we chose the following parameter set, which leads to an NMSSM Higgs and SUSY spectrum in accordance with the LHC data:

$$
\begin{aligned}
m_{\tilde{u}_{R}, \tilde{c}_{R}}=m_{\tilde{d}_{R}, \tilde{s}_{R}}=m_{\tilde{Q}_{1,2}}=m_{\tilde{L}_{1,2}}=m_{\tilde{e}_{R}, \tilde{\mu}_{R}}=3 \mathrm{TeV}, & m_{\tilde{t}_{R}}=748.07 \mathrm{GeV}, \\
m_{\tilde{Q}_{3}} & =1259 \mathrm{GeV}, \quad m_{\tilde{b}_{R}}=1709 \mathrm{GeV}, \quad m_{\tilde{L}_{3}}=1637 \mathrm{GeV}, \quad m_{\tilde{\tau}_{R}}=1618 \mathrm{GeV}, \\
A_{t}=1589 \mathrm{GeV}, \quad A_{u, c}=1675 \mathrm{GeV}, \quad A_{d, s, b} & =-669.04 \mathrm{GeV}, A_{e, \mu, \tau}=179.93 \mathrm{GeV}, \\
M_{1}=645.51 \mathrm{GeV}, \quad M_{2}=272.11 \mathrm{GeV}, \quad M_{3}=2511 \mathrm{GeV} &
\end{aligned}
$$

and the NMSSM specific input parameters

$$
\begin{aligned}
\lambda & =0.588, \quad \kappa \\
\tan \beta & =0.378, \quad A_{\kappa}=-675.73 \mathrm{GeV}, \quad \mu_{\mathrm{eff}}=385.90 \mathrm{GeV}, \\
M_{H^{ \pm}} & =639.08 \mathrm{GeV} .
\end{aligned}
$$

This results in the two-loop corrected mass $M_{A_{1}}$ of the lighter pseudoscalar $A_{1}$ and the stop masses $m_{\tilde{t}_{1,2}}$,

$$
M_{A_{1}}=637.40 \mathrm{GeV} \quad m_{\tilde{t}_{1}}=342.76 \mathrm{GeV} \quad \text { and } \quad m_{\tilde{t}_{2}}=1153 \mathrm{GeV} .
$$

And for the stop soft SUSY breaking trilinear coupling in the OS scheme we get

$$
A_{t}^{\mathrm{OS}}=1675 \mathrm{GeV} \text {. }
$$



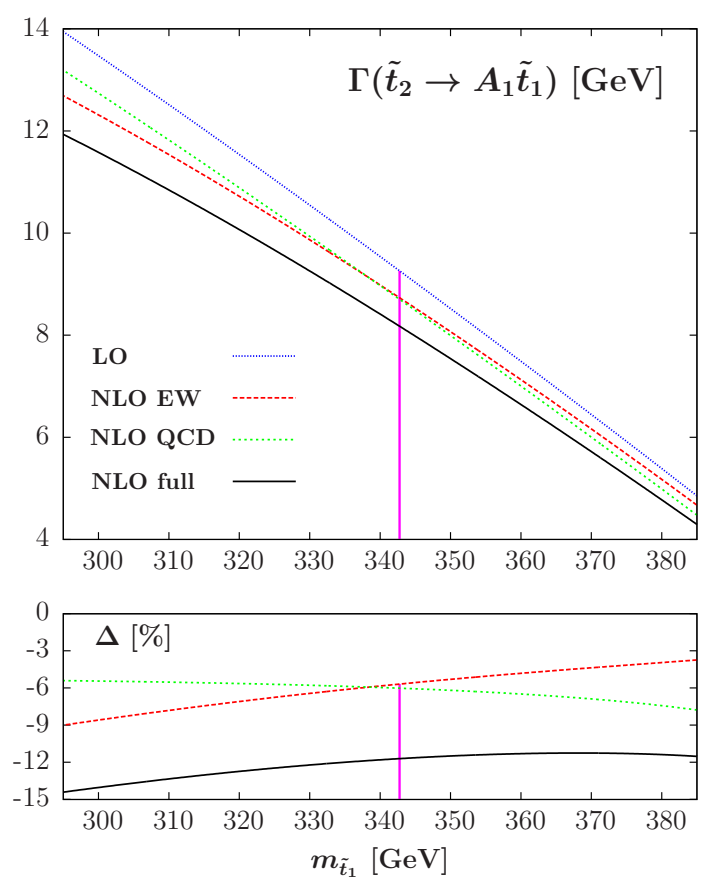

Figure 10. The decay width $\Gamma\left(\tilde{t}_{2} \rightarrow A_{1} \tilde{t}_{1}\right)$ as a function of $m_{\tilde{t}_{1}}$ at LO (blue/upper dotted), including the NLO QCD (green/lower dotted), the NLO EW (red/dashed) and both the EW and QCD corrections (black/full). Lower: the relative correction $\Delta=\left(\Gamma_{X}^{\mathrm{NLO}}-\Gamma^{\mathrm{LO}}\right) / \Gamma^{\mathrm{LO}}$ in per cent for $X=\mathrm{QCD}$ (green/dotted), EW (red/dashed) and the full NLO corrections (black/full). The pink line shows the position of the parameter point defined in eqs. (7.18)-(7.19).

Figure 10 (upper) shows the decay width $\Gamma\left(\tilde{t}_{2} \rightarrow A_{1} \tilde{t}_{1}\right)$ at LO, including the NLO EW, the NLO QCD and both NLO corrections, as a function of $m_{\tilde{t}_{1}}$, varied around the chosen parameter point, marked by the pink line in the plots. The LO decay width reaches

$$
\Gamma^{\mathrm{LO}}\left(\tilde{t}_{2} \rightarrow A_{1} \tilde{t}_{1}\right)=9.26 \mathrm{GeV} .
$$

In the whole investigated $m_{\tilde{t}_{1}}$ range, the EW and QCD corrections are significant, decreasing together the LO width by $\sim 12-15 \%$. Both corrections are of similar size, where, depending on the parameter point, once the QCD, once the EW corrections are more important. At our starting parameter point both corrections are almost equal resulting in a decrease of

$$
\Delta \Gamma^{\mathrm{QCD}+\mathrm{EW}}=-11.7 \%
$$

cf. figure 10 (lower). Our results demonstrate that also in the stop decays both the QCD and EW corrections have to be considered for a meaningful prediction of the stop decay width. 


\section{Conclusions}

The search for New Physics is one of the main tasks at the LHC. In the absence of any direct sign of new resonances so far, the precise investigation of the Higgs sector becomes more and more important. Physics beyond the SM might reveal itself in modified Higgs decay rates compared to the SM expectations or in the discovery of additional Higgs bosons, unambiguous sign of a non-SM Higgs sector. In view of the complexity of the experimental analyses and the plethora of still possible New Physics extensions, it is evident that the success of this research program depends on the precise predictions of parameters and observables from the theory side. In this paper we have calculated the NLO SUSY-EW and -QCD corrections to the decays of a pseudoscalar NMSSM Higgs boson into stop pairs and of the heavier stop into the lighter stop and a pseudoscalar Higgs boson. Both processes can become important in certain regions of the parameter space and hence contribute to the discovery channels of either the pseudoscalar Higgs boson and/or the stop quarks. The NLO corrections turn out to be important and, depending on the scenario, the EW corrections can be of same size as the QCD corrections and also come with opposite sign. Therefore not only the inclusion of higher order corrections is important but also the consideration of both the QCD and the EW corrections is indispensable for making reliable predictions.

\section{Acknowledgments}

JB, MM and KW have been supported in part by the DFG SFB/TR9 "Computational Particle Physics" and furthermore JB in part by the Institutional Strategy of the University of Tübingen (DFG, ZUK 63). The authors thank Michael Spira for helpful discussions.

\section{A The loop functions}

The $D$ dimensional one-loop integrals encountered in the calculation are the scalar one-, two- and three-point functions $A_{0}, B_{0}$ and $C_{0}$ as well as the coefficient of the two-point tensor integral of rank one, $B_{1}$. They are defined as

$$
\begin{aligned}
& A_{0}(m)=16 \pi^{2} \mu^{4-D} \int \frac{d^{D} q}{i(2 \pi)^{D}} \frac{1}{\left(q^{2}-m^{2}\right)} \\
& B_{0}\left(p^{2} ; m_{1}, m_{2}\right)= 16 \pi^{2} \mu^{4-D} \int \frac{d^{D} q}{i(2 \pi)^{D}} \frac{1}{\left(q^{2}-m_{1}^{2}\right)\left[(q+p)^{2}-m_{2}^{2}\right]} \\
& C_{0}\left(p_{1}^{2}, p_{2}^{2}, p_{12}^{2} ; m_{1}, m_{2}, m_{3}\right)= 16 \pi^{2} \mu^{4-D} \int \frac{d^{D} q}{i(2 \pi)^{D}} \\
& \times \frac{1}{\left(q^{2}-m_{1}^{2}\right)\left[\left(q+p_{1}\right)^{2}-m_{2}^{2}\right]\left[\left(q+p_{12}\right)^{2}-m_{3}^{2}\right]} \\
& p_{\mu} B_{1}\left(p^{2} ; m_{1}, m_{2}\right)= 16 \pi^{2} \mu^{4-D} \int \frac{d^{D} q}{i(2 \pi)^{D}} \frac{q_{\mu}}{\left(q^{2}-m_{1}^{2}\right)\left[(q+p)^{2}-m_{2}^{2}\right]},
\end{aligned}
$$

where

$$
p_{12} \equiv p_{1}+p_{2}
$$


with $p, p_{1}, p_{2}$ denoting the external momenta, which are taken as incoming, and $m, m_{1}$, $m_{2}, m_{3}$ the masses of the loop particles.

Open Access. This article is distributed under the terms of the Creative Commons Attribution License (CC-BY 4.0), which permits any use, distribution and reproduction in any medium, provided the original author(s) and source are credited.

\section{References}

[1] ATLAS collaboration, Observation of a new particle in the search for the Standard Model Higgs boson with the ATLAS detector at the LHC, Phys. Lett. B 716 (2012) 1 [arXiv:1207.7214] [INSPIRE].

[2] ATLAS collaboration, Updated ATLAS results on the signal strength of the Higgs-like boson for decays into $W W$ and heavy fermion final states, ATLAS-CONF-2012-162 (2012).

[3] CMS collaboration, Observation of a new boson at a mass of $125 \mathrm{GeV}$ with the CMS experiment at the LHC, Phys. Lett. B 716 (2012) 30 [arXiv:1207.7235] [INSPIRE].

[4] CMS collaboration, Combination of standard model Higgs boson searches and measurements of the properties of the new boson with a mass near 125 GeV, CMS-PAS-HIG-12-045 (2012).

[5] D.V. Volkov and V.P. Akulov, Is the Neutrino a Goldstone Particle?, Phys. Lett. B 46 (1973) 109 [INSPIRE].

[6] J. Wess and B. Zumino, Supergauge Transformations in Four-Dimensions, Nucl. Phys. B 70 (1974) 39 [InSPIRE].

[7] P. Fayet, Supersymmetry and Weak, Electromagnetic and Strong Interactions, Phys. Lett. B 64 (1976) 159 [InSPIRE].

[8] P. Fayet, Spontaneously Broken Supersymmetric Theories of Weak, Electromagnetic and Strong Interactions, Phys. Lett. B 69 (1977) 489 [INSPIRE].

[9] P. Fayet, Relations Between the Masses of the Superpartners of Leptons and Quarks, the Goldstino Couplings and the Neutral Currents, Phys. Lett. B 84 (1979) 416 [INSPIRE].

[10] G.R. Farrar and P. Fayet, Phenomenology of the Production, Decay and Detection of New Hadronic States Associated with Supersymmetry, Phys. Lett. B 76 (1978) 575 [InSPIRE].

[11] S. Dimopoulos and H. Georgi, Softly Broken Supersymmetry and SU(5), Nucl. Phys. B 193 (1981) 150 [InSPIRE].

[12] N. Sakai, Naturalness in Supersymmetric Guts, Z. Phys. C 11 (1981) 153 [InSPIRE].

[13] E. Witten, Dynamical Breaking of Supersymmetry, Nucl. Phys. B 188 (1981) 513 [INSPIRE].

[14] H.P. Nilles, Supersymmetry, Supergravity and Particle Physics, Phys. Rept. 110 (1984) 1 [INSPIRE].

[15] H.E. Haber and G.L. Kane, The Search for Supersymmetry: Probing Physics Beyond the Standard Model, Phys. Rept. 117 (1985) 75 [INSPIRE].

[16] M.F. Sohnius, Introducing Supersymmetry, Phys. Rept. 128 (1985) 39 [INSPIRE].

[17] J.F. Gunion and H.E. Haber, Higgs Bosons in Supersymmetric Models. 1., Nucl. Phys. B 272 (1986) 1 [Erratum ibid. B 402 (1993) 567] [InSPIRE]. 
[18] A.B. Lahanas and D.V. Nanopoulos, The Road to No Scale Supergravity, Phys. Rept. 145 (1987) 1 [INSPIRE].

[19] P. Fayet, Supergauge Invariant Extension of the Higgs Mechanism and a Model for the electron and Its Neutrino, Nucl. Phys. B 90 (1975) 104 [inSPIRE].

[20] R. Barbieri, S. Ferrara and C.A. Savoy, Gauge Models with Spontaneously Broken Local Supersymmetry, Phys. Lett. B 119 (1982) 343 [INSPIRE].

[21] M. Dine, W. Fischler and M. Srednicki, A Simple Solution to the Strong CP Problem with a Harmless Axion, Phys. Lett. B 104 (1981) 199 [INSPIRE].

[22] H.P. Nilles, M. Srednicki and D. Wyler, Weak Interaction Breakdown Induced by Supergravity, Phys. Lett. B 120 (1983) 346 [INSPIRE].

[23] J.M. Frere, D.R.T. Jones and S. Raby, Fermion Masses and Induction of the Weak Scale by Supergravity, Nucl. Phys. B 222 (1983) 11 [INSPIRE].

[24] J.P. Derendinger and C.A. Savoy, Quantum Effects and $\mathrm{SU}(2) \times \mathrm{U}(1)$ Breaking in Supergravity Gauge Theories, Nucl. Phys. B 237 (1984) 307 [InSPIRE].

[25] J.R. Ellis, J.F. Gunion, H.E. Haber, L. Roszkowski and F. Zwirner, Higgs Bosons in a Nonminimal Supersymmetric Model, Phys. Rev. D 39 (1989) 844 [INSPIRE].

[26] M. Drees, Supersymmetric Models with Extended Higgs Sector, Int. J. Mod. Phys. A 4 (1989) 3635 [inSPIRE].

[27] U. Ellwanger, M. Rausch de Traubenberg and C.A. Savoy, Particle spectrum in supersymmetric models with a gauge singlet, Phys. Lett. B 315 (1993) 331 [hep-ph/9307322] [INSPIRE].

[28] U. Ellwanger, M. Rausch de Traubenberg and C.A. Savoy, Phenomenology of supersymmetric models with a singlet, Nucl. Phys. B 492 (1997) 21 [hep-ph/9611251] [INSPIRE].

[29] T. Elliott, S.F. King and P.L. White, Unification constraints in the next-to-minimal supersymmetric standard model, Phys. Lett. B 351 (1995) 213 [hep-ph/9406303] [INSPIRE].

[30] S.F. King and P.L. White, Resolving the constrained minimal and next-to-minimal supersymmetric standard models, Phys. Rev. D 52 (1995) 4183 [hep-ph/9505326] [INSPIRE].

[31] F. Franke and H. Fraas, Neutralinos and Higgs bosons in the next-to-minimal supersymmetric standard model, Int. J. Mod. Phys. A 12 (1997) 479 [hep-ph/9512366] [INSPIRE].

[32] M. Maniatis, The Next-to-Minimal Supersymmetric extension of the Standard Model reviewed, Int. J. Mod. Phys. A 25 (2010) 3505 [arXiv:0906.0777] [InSPIRE].

[33] U. Ellwanger, C. Hugonie and A.M. Teixeira, The Next-to-Minimal Supersymmetric Standard Model, Phys. Rept. 496 (2010) 1 [arXiv:0910.1785] [InSPIRE].

[34] J.E. Kim and H.P. Nilles, The mu Problem and the Strong CP Problem, Phys. Lett. B 138 (1984) 150 [INSPIRE].

[35] M. Bastero-Gil, C. Hugonie, S.F. King, D.P. Roy and S. Vempati, Does LEP prefer the NMSSM?, Phys. Lett. B 489 (2000) 359 [hep-ph/0006198] [INSPIRE]. 
[36] A. Delgado, C. Kolda, J.P. Olson and A. de la Puente, Solving the Little Hierarchy Problem with a Singlet and Explicit $\mu$ Terms, Phys. Rev. Lett. 105 (2010) 091802 [arXiv: 1005.1282] [INSPIRE].

[37] U. Ellwanger, G. Espitalier-Noel and C. Hugonie, Naturalness and Fine Tuning in the NMSSM: Implications of Early LHC Results, JHEP 09 (2011) 105 [arXiv:1107.2472] [INSPIRE].

[38] G.G. Ross and K. Schmidt-Hoberg, The Fine-Tuning of the Generalised NMSSM, Nucl. Phys. B 862 (2012) 710 [arXiv:1108.1284] [INSPIRE].

[39] L.J. Hall, D. Pinner and J.T. Ruderman, A Natural SUSY Higgs Near 126 GeV, JHEP 04 (2012) 131 [arXiv:1112.2703] [INSPIRE].

[40] Z. Kang, J. Li and T. Li, On Naturalness of the MSSM and NMSSM, JHEP 11 (2012) 024 [arXiv:1201.5305] [INSPIRE].

[41] J.-J. Cao, Z.-X. Heng, J.M. Yang, Y.-M. Zhang and J.-Y. Zhu, A SM-like Higgs near $125 \mathrm{GeV}$ in low energy SUSY: a comparative study for MSSM and NMSSM, JHEP 03 (2012) 086 [arXiv:1202.5821] [INSPIRE].

[42] J. Cao, Z. Heng, J.M. Yang and J. Zhu, Status of low energy SUSY models confronted with the LHC $125 \mathrm{GeV}$ Higgs data, JHEP 10 (2012) 079 [arXiv:1207.3698] [INSPIRE].

[43] J.R. Espinosa, C. Grojean, V. Sanz and M. Trott, NSUSY fits, JHEP 12 (2012) 077 [arXiv:1207.7355] [INSPIRE].

[44] M. Perelstein and B. Shakya, XENON100 implications for naturalness in the MSSM, NMSSM and $\lambda$-supersymmetry model, Phys. Rev. D 88 (2013) 075003 [arXiv:1208.0833] [INSPIRE].

[45] K. Agashe, Y. Cui and R. Franceschini, Natural Islands for a $125 \mathrm{GeV}$ Higgs in the scale-invariant NMSSM, JHEP 02 (2013) 031 [arXiv:1209.2115] [INSPIRE].

[46] S.F. King, M. Mühlleitner and R. Nevzorov, NMSSM Higgs Benchmarks Near $125 \mathrm{GeV}$, Nucl. Phys. B 860 (2012) 207 [arXiv:1201.2671] [INSPIRE].

[47] S.F. King, M. Mühlleitner, R. Nevzorov and K. Walz, Natural NMSSM Higgs Bosons, Nucl. Phys. B 870 (2013) 323 [arXiv:1211.5074] [INSPIRE].

[48] S.F. King, M. Mühlleitner, R. Nevzorov and K. Walz, Discovery Prospects for NMSSM Higgs Bosons at the High-Energy Large Hadron Collider, Phys. Rev. D 90 (2014) 095014 [arXiv:1408.1120] [INSPIRE].

[49] U. Ellwanger, Enhanced di-photon Higgs signal in the Next-to-Minimal Supersymmetric Standard Model, Phys. Lett. B 698 (2011) 293 [arXiv:1012.1201] [InSPIRE].

[50] U. Ellwanger, A Higgs boson near $125 \mathrm{GeV}$ with enhanced di-photon signal in the NMSSM, JHEP 03 (2012) 044 [arXiv:1112.3548] [INSPIRE].

[51] A. Arvanitaki and G. Villadoro, A Non Standard Model Higgs at the LHC as a Sign of Naturalness, JHEP 02 (2012) 144 [arXiv:1112.4835] [INSPIRE].

[52] J.F. Gunion, Y. Jiang and S. Kraml, Could two NMSSM Higgs bosons be present near 125 GeV?, Phys. Rev. D 86 (2012) 071702 [arXiv:1207.1545] [INSPIRE].

[53] J.F. Gunion, Y. Jiang and S. Kraml, Diagnosing Degenerate Higgs Bosons at $125 \mathrm{GeV}$, Phys. Rev. Lett. 110 (2013) 051801 [arXiv:1208.1817] [InSPIRE]. 
[54] C. Han, X. Ji, L. Wu, P. Wu and J.M. Yang, Higgs pair production with SUSY QCD correction: revisited under current experimental constraints, JHEP 04 (2014) 003 [arXiv: 1307.3790] [INSPIRE].

[55] Z. Kang, J. Li, T. Li, D. Liu and J. Shu, Probing the CP-even Higgs sector via $\mathrm{H}_{3} \rightarrow \mathrm{H}_{2} \mathrm{H}_{1}$ in the natural next-to-minimal supersymmetric standard model, Phys. Rev. D 88 (2013) 015006 [arXiv:1301.0453] [inSPIRE].

[56] D.G. Cerdeno, P. Ghosh and C.B. Park, Probing the two light Higgs scenario in the NMSSM with a low-mass pseudoscalar, JHEP 06 (2013) 031 [arXiv:1301.1325] [INSPIRE].

[57] M. Badziak, M. Olechowski and S. Pokorski, New Regions in the NMSSM with a $125 \mathrm{GeV}$ Higgs, JHEP 06 (2013) 043 [arXiv: 1304.5437] [INSPIRE].

[58] S. Munir, L. Roszkowski and S. Trojanowski, Simultaneous enhancement in $\gamma \gamma, b \bar{b}$ and $\tau^{+} \tau^{-}$rates in the NMSSM with nearly degenerate scalar and pseudoscalar Higgs bosons, Phys. Rev. D 88 (2013) 055017 [arXiv:1305.0591] [INSPIRE].

[59] D.T. Nhung, M. Mühlleitner, J. Streicher and K. Walz, Higher Order Corrections to the Trilinear Higgs Self-Couplings in the Real NMSSM, JHEP 11 (2013) 181 [arXiv:1306.3926] [INSPIRE].

[60] U. Ellwanger, Higgs pair production in the NMSSM at the LHC, JHEP 08 (2013) 077 [arXiv:1306.5541] [INSPIRE].

[61] D.G. Cerdeño, P. Ghosh, C.B. Park and M. Peiró, Collider signatures of a light NMSSM pseudoscalar in neutralino decays in the light of LHC results, JHEP 02 (2014) 048 [arXiv: 1307.7601] [INSPIRE].

[62] C. Beskidt, W. de Boer and D.I. Kazakov, A comparison of the Higgs sectors of the CMSSM and NMSSM for a $126 \mathrm{GeV}$ Higgs boson, Phys. Lett. B 726 (2013) 758 [arXiv:1308.1333] [INSPIRE].

[63] K. Choi, S.H. Im, K.S. Jeong and M.-S. Seo, Higgs phenomenology in the Peccei-Quinn invariant NMSSM, JHEP 01 (2014) 072 [arXiv: 1308.4447] [INSPIRE].

[64] J. Kozaczuk and S. Profumo, Light NMSSM neutralino dark matter in the wake of CDMS II and a 126 GeV Higgs boson, Phys. Rev. D 89 (2014) 095012 [arXiv:1308.5705] [INSPIRE].

[65] J. Cao, F. Ding, C. Han, J.M. Yang and J. Zhu, A light Higgs scalar in the NMSSM confronted with the latest LHC Higgs data, JHEP 11 (2013) 018 [arXiv:1309.4939] [INSPIRE].

[66] J.-W. Fan et al., Study of diphoton decays of the lightest scalar Higgs boson in the Next-to-Minimal Supersymmetric Standard Model, Chin. Phys. C 38 (2014) 073101 [arXiv: 1309.6394] [INSPIRE].

[67] J. Huang, T. Liu, L.-T. Wang and F. Yu, Supersymmetric Exotic Decays of the $125 \mathrm{GeV}$ Higgs Boson, Phys. Rev. Lett. 112 (2014) 221803 [arXiv:1309.6633] [INSPIRE].

[68] S. Munir, Novel Higgs-to-125 GeV Higgs boson decays in the complex NMSSM, Phys. Rev. D 89 (2014) 095013 [arXiv:1310.8129] [INSPIRE].

[69] G. Bélanger, V. Bizouard and G. Chalons, Boosting Higgs boson decays into gamma and a $Z$ in the NMSSM, Phys. Rev. D 89 (2014) 095023 [arXiv: 1402.3522] [INSPIRE].

[70] C. Beskidt, W. de Boer and D.I. Kazakov, The impact of a $126 \mathrm{GeV}$ Higgs on the neutralino mass, Phys. Lett. B 738 (2014) 505 [arXiv:1402.4650] [INSPIRE]. 
[71] U. Ellwanger and C. Hugonie, The semi-constrained NMSSM satisfying bounds from the LHC, LUX and Planck, JHEP 08 (2014) 046 [arXiv: 1405.6647] [INSPIRE].

[72] U. Ellwanger and A.M. Teixeira, NMSSM with a singlino LSP: possible challenges for searches for supersymmetry at the LHC, JHEP 10 (2014) 113 [arXiv:1406.7221] [INSPIRE].

[73] D. Das, L. Mitzka and W. Porod, Discovery of Charged Higgs through $\gamma \gamma$ final states, arXiv: 1408.1704 [INSPIRE].

[74] J. Cao, D. Li, L. Shang, P. Wu and Y. Zhang, Exploring the Higgs Sector of a Most Natural NMSSM and its Prediction on Higgs Pair Production at the LHC, JHEP 12 (2014) 026 [arXiv:1409.8431] [INSPIRE].

[75] N.-E. Bomark, S. Moretti, S. Munir and L. Roszkowski, A light NMSSM pseudoscalar Higgs boson at the LHC redux, JHEP 02 (2015) 044 [arXiv: 1409.8393] [INSPIRE].

[76] U. Ellwanger and A.M. Teixeira, Excessive Higgs pair production with little MET from squarks and gluinos in the NMSSM, JHEP 04 (2015) 172 [arXiv:1412.6394] [INSPIRE].

[77] L. Wu, J.M. Yang, C.-P. Yuan and M. Zhang, Higgs self-coupling in the MSSM and NMSSM after the LHC Run 1, Phys. Lett. B 747 (2015) 378 [arXiv:1504.06932] [InSPIRE].

[78] S. Moretti and S. Munir, Two Higgs bosons near $125 \mathrm{GeV}$ in the complex NMSSM and the LHC Run-I data, arXiv:1505.00545 [INSPIRE].

[79] D. Buttazzo, F. Sala and A. Tesi, Singlet-like Higgs bosons at present and future colliders, arXiv: 1505.05488 [INSPIRE].

[80] C.T. Potter, Natural NMSSM with a Light Pseudoscalar Higgs and Singlino LSP, arXiv: 1505.05554 [INSPIRE].

[81] A. Djouadi, J. Kalinowski, P. Ohmann and P.M. Zerwas, Heavy SUSY Higgs bosons at $e^{+} e^{-}$linear colliders, Z. Phys. C 74 (1997) 93 [hep-ph/9605339] [InSPIRE].

[82] A. Djouadi, P. Janot, J. Kalinowski and P.M. Zerwas, SUSY decays of Higgs particles, Phys. Lett. B 376 (1996) 220 [hep-ph/9603368] [INSPIRE].

[83] A. Bartl, H. Eberl, K. Hidaka, T. Kon, W. Majerotto and Y. Yamada, QCD corrections to Higgs boson decays into squarks in the minimal supersymmetric standard model, Phys. Lett. B 402 (1997) 303 [hep-ph/9701398] [INSPIRE].

[84] A. Arhrib, A. Djouadi, W. Hollik and C. Junger, SUSY Higgs boson decays into scalar quarks: QCD corrections, Phys. Rev. D 57 (1998) 5860 [hep-ph/9702426] [INSPIRE].

[85] H. Eberl, K. Hidaka, S. Kraml, W. Majerotto and Y. Yamada, Improved SUSY QCD corrections to Higgs boson decays into quarks and squarks, Phys. Rev. D 62 (2000) 055006 [hep-ph/9912463] [INSPIRE].

[86] E. Accomando, G. Chachamis, F. Fugel, M. Spira and M. Walser, Neutral Higgs Boson Decays to Squark Pairs reanalyzed, Phys. Rev. D 85 (2012) 015004 [arXiv:1103.4283] [INSPIRE].

[87] C. Weber, H. Eberl and W. Majerotto, Improved full one loop corrections to $A^{0} \rightarrow \tilde{q}_{1} \overline{\tilde{q}}_{2}$ and $\tilde{q}_{2} \rightarrow \tilde{q}_{1} A^{0}$, Phys. Lett. B 572 (2003) 56 [hep-ph/0305250] [INSPIRE].

[88] C. Weber, H. Eberl and W. Majerotto, Improved full one loop corrections to $A^{0} \rightarrow \tilde{f}_{1} \overline{\tilde{f}}_{2}$ and $\tilde{f}_{2} \rightarrow \tilde{f}_{1} A^{0}$, Phys. Rev. D 68 (2003) 093011 [hep-ph/0308146] [INSPIRE]. 
[89] A. Bartl et al., Bosonic decays of $\tilde{t}_{2}$ and $\tilde{b}_{2}$, Phys. Lett. B 435 (1998) 118 [hep-ph/9804265] [INSPIRE].

[90] A. Bartl et al., SUSY-QCD corrections to top and bottom squark decays into Higgs bosons, Phys. Rev. D 59 (1999) 115007 [hep-ph/9806299] [inSPIRE].

[91] S. Heinemeyer, H. Rzehak and C. Schappacher, Proposals for Bottom Quark/Squark Renormalization in the Complex MSSM, Phys. Rev. D 82 (2010) 075010 [arXiv: 1007.0689] [INSPIRE].

[92] T. Fritzsche, S. Heinemeyer, H. Rzehak and C. Schappacher, Heavy Scalar Top Quark Decays in the Complex MSSM: A Full One-Loop Analysis, Phys. Rev. D 86 (2012) 035014 [arXiv: 1111.7289] [INSPIRE].

[93] S. Heinemeyer and C. Schappacher, Heavy Higgs Decays into Sfermions in the Complex MSSM: A Full One-Loop Analysis, Eur. Phys. J. C 75 (2015) 198 [arXiv:1410.2787] [INSPIRE].

[94] M. Krämer and M. Mühlleitner, Higgs Physics, Nucl. Part. Phys. Proc. 261-262 (2015) 246 [arXiv:1501.06658] [InSPIRE].

[95] U. Ellwanger, Radiative corrections to the neutral Higgs spectrum in supersymmetry with a gauge singlet, Phys. Lett. B 303 (1993) 271 [hep-ph/9302224] [INSPIRE].

[96] T. Elliott, S.F. King and P.L. White, Supersymmetric Higgs bosons at the limit, Phys. Lett. B 305 (1993) 71 [hep-ph/9302202] [INSPIRE].

[97] T. Elliott, S.F. King and P.L. White, Squark contributions to Higgs boson masses in the next-to-minimal supersymmetric standard model, Phys. Lett. B 314 (1993) 56 [hep-ph/9305282] [INSPIRE].

[98] T. Elliott, S.F. King and P.L. White, Radiative corrections to Higgs boson masses in the next-to-minimal supersymmetric Standard Model, Phys. Rev. D 49 (1994) 2435 [hep-ph/9308309] [INSPIRE].

[99] P.N. Pandita, Radiative corrections to the scalar Higgs masses in a nonminimal supersymmetric Standard Model, Z. Phys. C 59 (1993) 575 [inSPIRE].

[100] U. Ellwanger and C. Hugonie, Yukawa induced radiative corrections to the lightest Higgs boson mass in the NMSSM, Phys. Lett. B 623 (2005) 93 [hep-ph/0504269] [InSPIRE].

[101] G. Degrassi and P. Slavich, On the radiative corrections to the neutral Higgs boson masses in the NMSSM, Nucl. Phys. B 825 (2010) 119 [arXiv:0907.4682] [INSPIRE].

[102] F. Staub, W. Porod and B. Herrmann, The electroweak sector of the NMSSM at the one-loop level, JHEP 10 (2010) 040 [arXiv: 1007.4049] [INSPIRE].

[103] K. Ender, T. Graf, M. Mühlleitner and H. Rzehak, Analysis of the NMSSM Higgs Boson Masses at One-Loop Level, Phys. Rev. D 85 (2012) 075024 [arXiv:1111.4952] [INSPIRE].

[104] M.D. Goodsell, K. Nickel and F. Staub, Two-loop corrections to the Higgs masses in the NMSSM, Phys. Rev. D 91 (2015) 035021 [arXiv:1411.4665] [INSPIRE].

[105] M. Goodsell, K. Nickel and F. Staub, Generic two-loop Higgs mass calculation from a diagrammatic approach, Eur. Phys. J. C 75 (2015) 290 [arXiv:1503.03098] [INSPIRE].

[106] U. Ellwanger, J.F. Gunion and C. Hugonie, NMHDECAY: A Fortran code for the Higgs masses, couplings and decay widths in the NMSSM, JHEP 02 (2005) 066 [hep-ph/0406215] [INSPIRE]. 
[107] U. Ellwanger and C. Hugonie, NMHDECAY 2.0: An updated program for sparticle masses, Higgs masses, couplings and decay widths in the NMSSM,

Comput. Phys. Commun. 175 (2006) 290 [hep-ph/0508022] [INSPIRE].

[108] U. Ellwanger and C. Hugonie, NMSPEC: A Fortran code for the sparticle and Higgs masses in the NMSSM with GUT scale boundary conditions, Comput. Phys. Commun. 177 (2007) 399 [hep-ph/0612134] [INSPIRE].

[109] B.C. Allanach, SOFTSUSY: a program for calculating supersymmetric spectra, Comput. Phys. Commun. 143 (2002) 305 [hep-ph/0104145] [INSPIRE].

[110] B.C. Allanach, P. Athron, L.C. Tunstall, A. Voigt and A.G. Williams, Next-to-Minimal SOFTSUSY, Comput. Phys. Commun. 185 (2014) 2322 [arXiv:1311.7659] [InSPIRE].

[111] F. Staub, Automatic Calculation of supersymmetric Renormalization Group Equations and Self Energies, Comput. Phys. Commun. 182 (2011) 808 [arXiv:1002.0840] [INSPIRE].

[112] F. Staub, SARAH 3.2: Dirac Gauginos, UFO output and more, Comput. Phys. Commun. 184 (2013) pp. 1792-1809 [arXiv:1207.0906] [INSPIRE].

[113] F. Staub, SARAH 4: A tool for (not only SUSY) model builders, Comput. Phys. Commun. 185 (2014) 1773 [arXiv:1309.7223] [INSPIRE].

[114] M.D. Goodsell, K. Nickel and F. Staub, Two-Loop Higgs mass calculations in supersymmetric models beyond the MSSM with SARAH and SPheno, Eur. Phys. J. C 75 (2015) 32 [arXiv:1411.0675] [InSPIRE].

[115] W. Porod, SPheno, a program for calculating supersymmetric spectra, SUSY particle decays and SUSY particle production at $e^{+} e^{-}$colliders, Comput. Phys. Commun. 153 (2003) 275 [hep-ph/0301101] [INSPIRE].

[116] W. Porod and F. Staub, SPheno 3.1: Extensions including flavour, CP-phases and models beyond the MSSM, Comput. Phys. Commun. 183 (2012) 2458 [arXiv:1104.1573] [INSPIRE].

[117] P. Athron, J.-h. Park, D. Stöckinger and A. Voigt, FlexibleSUSY - A spectrum generator generator for supersymmetric models, Comput. Phys. Commun. 190 (2015) 139 [arXiv: 1406.2319] [INSPIRE].

[118] P. Athron, J.-h. Park, D. Stöckinger and A. Voigt, FlexibleSUSY - a meta spectrum generator for supersymmetric models, arXiv:1410.7385 [INSPIRE].

[119] F. Domingo, A New Tool for the study of the CP-violating NMSSM, JHEP 06 (2015) 052 [arXiv: 1503.07087] [INSPIRE].

[120] J. Baglio et al., NMSSMCALC: A Program Package for the Calculation of Loop-Corrected Higgs Boson Masses and Decay Widths in the (Complex) NMSSM, Comput. Phys. Commun. 185 (2014) 3372 [arXiv:1312.4788] [InSPIRE].

[121] T. Graf, R. Grober, M. Mühlleitner, H. Rzehak and K. Walz, Higgs Boson Masses in the Complex NMSSM at One-Loop Level, JHEP 10 (2012) 122 [arXiv:1206.6806] [INSPIRE].

[122] M. Mühlleitner, D.T. Nhung, H. Rzehak and K. Walz, Two-loop contributions of the order $\mathcal{O}\left(\alpha_{t} \alpha_{s}\right)$ to the masses of the Higgs bosons in the CP-violating NMSSM, JHEP 05 (2015) 128 [arXiv: 1412.0918] [INSPIRE].

[123] S. Liebler, Neutral Higgs production at proton colliders in the CP-conserving NMSSM, Eur. Phys. J. C 75 (2015) 210 [arXiv:1502.07972] [INSPIRE]. 
[124] M. Frank, T. Hahn, S. Heinemeyer, W. Hollik, H. Rzehak and G. Weiglein, The Higgs Boson Masses and Mixings of the Complex MSSM in the Feynman-Diagrammatic Approach, JHEP 02 (2007) 047 [hep-ph/0611326] [INSPIRE].

[125] W. Siegel, Supersymmetric Dimensional Regularization via Dimensional Reduction, Phys. Lett. B 84 (1979) 193 [inSPIRE].

[126] D.M. Capper, D.R.T. Jones and P. van Nieuwenhuizen, Regularization by Dimensional Reduction of Supersymmetric and Nonsupersymmetric Gauge Theories,

Nucl. Phys. B 167 (1980) 479 [inSPIRE].

[127] G. 't Hooft and M.J.G. Veltman, Scalar One Loop Integrals, Nucl. Phys. B 153 (1979) 365 [INSPIRE].

[128] G. Passarino and M.J.G. Veltman, One Loop Corrections for $e^{+} e^{-}$Annihilation Into $\mu^{+} \mu^{-}$ in the Weinberg Model, Nucl. Phys. B 160 (1979) 151 [InSPIRE].

[129] T. Hahn, Generating Feynman diagrams and amplitudes with FeynArts 3 , Comput. Phys. Commun. 140 (2001) 418 [hep-ph/0012260] [INSPIRE].

[130] T. Hahn and C. Schappacher, The implementation of the minimal supersymmetric standard model in FeynArts and FormCalc, Comput. Phys. Commun. 143 (2002) 54 [hep-ph/0105349] [INSPIRE].

[131] T. Hahn and M. Pérez-Victoria, Automatized one loop calculations in four-dimensions and D-dimensions, Comput. Phys. Commun. 118 (1999) 153 [hep-ph/9807565] [INSPIRE].

[132] T. Hahn, A Mathematica interface for FormCalc-generated code, Comput. Phys. Commun. 178 (2008) 217 [hep-ph/0611273] [INSPIRE].

[133] A. Djouadi, J. Kalinowski and M. Spira, HDECAY: A program for Higgs boson decays in the standard model and its supersymmetric extension, Comput. Phys. Commun. 108 (1998) 56 [hep-ph/9704448] [INSPIRE].

[134] J.M. Butterworth et al., The Tools and Monte Carlo Working Group Summary Report from the Les Houches 2009 Workshop on TeV Colliders, arXiv:1003.1643 [INSPIRE].

[135] A. Djouadi, M.M. Mühlleitner and M. Spira, Decays of supersymmetric particles: The program SUSY-HIT (SUspect-SdecaY-HDECAY-InTerface), Acta Phys. Polon. B 38 (2007) 635 [hep-ph/0609292] [INSPIRE].

[136] M. Mühlleitner, A. Djouadi and Y. Mambrini, SDECAY: A Fortran code for the decays of the supersymmetric particles in the MSSM, Comput. Phys. Commun. 168 (2005) 46 [hep-ph/0311167] [INSPIRE].

[137] M. Mühlleitner, SDECAY: A Fortran code for SUSY particle decays in the MSSM, Acta Phys. Polon. B 35 (2004) 2753 [hep-ph/0409200] [INSPIRE].

[138] A. Dabelstein, The one loop renormalization of the MSSM Higgs sector and its application to the neutral scalar Higgs masses, Z. Phys. C 67 (1995) 495 [hep-ph/9409375] [InSPIRE].

[139] A. Dabelstein, Fermionic decays of neutral MSSM Higgs bosons at the one loop level, Nucl. Phys. B 456 (1995) 25 [hep-ph/9503443] [INSPIRE].

[140] P. Bechtle, O. Brein, S. Heinemeyer, G. Weiglein and K.E. Williams, HiggsBounds: Confronting Arbitrary Higgs Sectors with Exclusion Bounds from LEP and the Tevatron, Comput. Phys. Commun. 181 (2010) 138 [arXiv:0811.4169] [INSPIRE]. 
[141] P. Bechtle, O. Brein, S. Heinemeyer, G. Weiglein and K.E. Williams, HiggsBounds 2.0.0: Confronting Neutral and Charged Higgs Sector Predictions with Exclusion Bounds from LEP and the Tevatron, Comput. Phys. Commun. 182 (2011) 2605 [arXiv:1102.1898] [INSPIRE].

[142] P. Bechtle et al., HiggsBounds-4: Improved Tests of Extended Higgs Sectors against Exclusion Bounds from LEP, the Tevatron and the LHC, Eur. Phys. J. C 74 (2014) 2693 [arXiv:1311.0055] [INSPIRE].

[143] P. Bechtle, S. Heinemeyer, O. Stål, T. Stefaniak and G. Weiglein, HiggsSignals: Confronting arbitrary Higgs sectors with measurements at the Tevatron and the LHC, Eur. Phys. J. C 74 (2014) 2711 [arXiv:1305.1933] [INSPIRE].

[144] T. Inami, T. Kubota and Y. Okada, Effective Gauge Theory and the Effect of Heavy Quarks in Higgs Boson Decays, Z. Phys. C 18 (1983) 69 [inSPIRE].

[145] A. Djouadi, M. Spira and P.M. Zerwas, Production of Higgs bosons in proton colliders: QCD corrections, Phys. Lett. B 264 (1991) 440 [inSPIRE].

[146] M. Spira, A. Djouadi, D. Graudenz and P.M. Zerwas, SUSY Higgs production at proton colliders, Phys. Lett. B 318 (1993) 347 [INSPIRE].

[147] M. Spira, A. Djouadi, D. Graudenz and P.M. Zerwas, Higgs boson production at the LHC, Nucl. Phys. B 453 (1995) 17 [hep-ph/9504378] [INSPIRE].

[148] M. Krämer, E. Laenen and M. Spira, Soft gluon radiation in Higgs boson production at the LHC, Nucl. Phys. B 511 (1998) 523 [hep-ph/9611272] [INSPIRE].

[149] K.G. Chetyrkin, B.A. Kniehl and M. Steinhauser, Hadronic Higgs decay to order $\alpha_{S}^{4}$, Phys. Rev. Lett. 79 (1997) 353 [hep-ph/9705240] [INSPIRE].

[150] K.G. Chetyrkin, B.A. Kniehl and M. Steinhauser, Decoupling relations to $\mathcal{O}\left(\alpha_{S}^{3}\right)$ and their connection to low-energy theorems, Nucl. Phys. B 510 (1998) 61 [hep-ph/9708255] [INSPIRE].

[151] Y. Schröder and M. Steinhauser, Four-loop decoupling relations for the strong coupling, JHEP 01 (2006) 051 [hep-ph/0512058] [INSPIRE].

[152] K.G. Chetyrkin, J.H. Kuhn and C. Sturm, QCD decoupling at four loops, Nucl. Phys. B 744 (2006) 121 [hep-ph/0512060] [rNSPIRE].

[153] P.A. Baikov and K.G. Chetyrkin, Top Quark Mediated Higgs Boson Decay into Hadrons to Order $\alpha_{s}^{5}$, Phys. Rev. Lett. 97 (2006) 061803 [hep-ph/0604194] [INSPIRE].

[154] S. Dawson, A. Djouadi and M. Spira, QCD corrections to SUSY Higgs production: The role of squark loops, Phys. Rev. Lett. 77 (1996) 16 [hep-ph/9603423] [INSPIRE].

[155] A. Djouadi, V. Driesen, W. Hollik and J.I. Illana, The coupling of the lightest SUSY Higgs boson to two photons in the decoupling regime, Eur. Phys. J. C 1 (1998) 149 [hep-ph/9612362] [INSPIRE]. 
[156] ATLAS collaboration, Search for a supersymmetric partner to the top quark in final states with jets and missing transverse momentum at $\sqrt{s}=7 \mathrm{TeV}$ with the ATLAS detector, Phys. Rev. Lett. 109 (2012) 211802 [arXiv:1208.1447] [INSPIRE].

[157] ATLAS collaboration, Search for direct top squark pair production in final states with one isolated lepton, jets and missing transverse momentum in $\sqrt{s}=7$ TeV pp collisions using $4.7 \mathrm{fb}^{-1}$ of ATLAS data, Phys. Rev. Lett. 109 (2012) 211803 [arXiv:1208.2590] [InSPIRE].

[158] ATLAS collaboration, Search for a heavy top-quark partner in final states with two leptons with the ATLAS detector at the LHC, JHEP 11 (2012) 094 [arXiv: 1209.4186] [INSPIRE].

[159] ATLAS collaboration, Search for direct top-squark pair production in final states with two leptons in pp collisions at $\sqrt{s}=8 \mathrm{TeV}$ with the ATLAS detector, JHEP 06 (2014) 124 [arXiv: 1403.4853] [INSPIRE].

[160] ATLAS collaboration, Search for direct pair production of the top squark in all-hadronic final states in proton-proton collisions at $\sqrt{s}=8 \mathrm{TeV}$ with the ATLAS detector, JHEP 09 (2014) 015 [arXiv:1406.1122] [INSPIRE].

[161] ATLAS collaboration, Measurement of Spin Correlation in Top-Antitop Quark Events and Search for Top Squark Pair Production in pp Collisions at $\sqrt{s}=8 \mathrm{TeV}$ Using the ATLAS Detector, Phys. Rev. Lett. 114 (2015) 142001 [arXiv:1412.4742] [INSPIRE].

[162] ATLAS collaboration, Search for top squark pair production in final states with one isolated lepton, jets and missing transverse momentum in $\sqrt{s}=8 \mathrm{TeV}$ pp collisions with the ATLAS detector, JHEP 11 (2014) 118 [arXiv:1407.0583] [INSPIRE].

[163] ATLAS collaboration, Search for pair-produced third-generation squarks decaying via charm quarks or in compressed supersymmetric scenarios in pp collisions at $\sqrt{s}=8 \mathrm{TeV}$ with the ATLAS detector, Phys. Rev. D 90 (2014) 052008 [arXiv:1407.0608] [InSPIRE].

[164] CMS collaboration, Search for top-squark pair production in the single-lepton final state in pp collisions at $\sqrt{s}=8 \mathrm{TeV}$, Eur. Phys. J. C 73 (2013) 2677 [arXiv:1308.1586] [INSPIRE].

[165] CMS collaboration, Search for top squark and higgsino production using diphoton Higgs boson decays, Phys. Rev. Lett. 112 (2014) 161802 [arXiv:1312.3310] [INSPIRE].

[166] CMS collaboration, Search for supersymmetry using razor variables in events with b-tagged jets in pp collisions at $\sqrt{s}=8 \mathrm{TeV}$, Phys. Rev. D 91 (2015) 052018 [arXiv:1502.00300] [INSPIRE].

[167] CMS collaboration, Search for top squarks in multijet events with large missing momentum in proton-proton collisions at $8 \mathrm{TeV}$, CMS-PAS-SUS-13-015 (2013).

[168] CMS collaboration, Search for top-squark pair production with Higgs and $Z$ bosons in the final state in pp collisions at 8 TeV, CMS-PAS-SUS-13-024 (2014).

[169] CMS collaboration, Exclusion limits on gluino and top-squark pair production in natural SUSY scenarios with inclusive razor and exclusive single-lepton searches at $8 \mathrm{TeV}$, CMS-PAS-SUS-14-011 (2014).

[170] CMS collaboration, Search for top squarks decaying to a charm quark and a neutralino in events with a jet and missing transverse momentum, CMS-PAS-SUS-13-009 (2014).

[171] Particle Data Group collaboration, K.A. Olive et al. Review of Particle Physics, Chin. Phys. C 38 (2014) 090001. 
[172] F. Jegerlehner, Electroweak effective couplings for future precision experiments, Nuovo Cim. C 034S1 (2011) 31 [arXiv:1107.4683] [INSPIRE].

[173] P.Z. Skands et al., SUSY Les Houches accord: Interfacing SUSY spectrum calculators, decay packages and event generators, JHEP 07 (2004) 036 [hep-ph/0311123] [INSPIRE].

[174] B.C. Allanach et al., SUSY Les Houches Accord 2, Comput. Phys. Commun. 180 (2009) 8 [arXiv:0801.0045] [INSPIRE].

[175] M. Mühlleitner and E. Popenda, Light Stop Decay in the MSSM with Minimal Flavour Violation, JHEP 04 (2011) 095 [arXiv: 1102.5712] [INSPIRE].

[176] R. Gröber, M.M. Mühlleitner, E. Popenda and A. Wlotzka, Light Stop Decays: Implications for LHC Searches, Eur. Phys. J. C 75 (2015) 420 [arXiv:1408.4662] [INSPIRE].

[177] D. Ghosh, Boosted dibosons from mixed heavy top squarks, Phys. Rev. D 88 (2013) 115013 [arXiv: 1308.0320] [INSPIRE].

[178] A. Chakraborty, D.K. Ghosh, S. Mondal, S. Poddar and D. Sengupta, Probing the NMSSM via Higgs boson signatures from stop cascade decays at the LHC, Phys. Rev. D 91 (2015) 115018 [arXiv: 1503.07592] [INSPIRE]. 